\title{
The CPA Equation of State and an Activity Coefficient Model for Accurate Molar Enthalpy Calculations of Mixtures with Carbon Dioxide and Water/Brine
}

\author{
Philip C. Myint ${ }^{1,2, *}$, Yue $\mathrm{Hao}^{2, \dagger}$, and Abbas Firoozabadi ${ }^{1,3, \ddagger}$ \\ ${ }^{1}$ Department of Chemical and Environmental Engineering, Yale University \\ ${ }^{2}$ Lawrence Livermore National Laboratory, Livermore, CA \\ ${ }^{3}$ Reservoir Engineering Research Institute, Palo Alto, CA
}

April 21, 2015

\begin{abstract}
Thermodynamic property calculations of mixtures containing carbon dioxide $\left(\mathrm{CO}_{2}\right)$ and water, including brines, are essential in theoretical models of many natural and industrial processes. The properties of greatest practical interest are density, solubility, and enthalpy. Many models for density and solubility calculations have been presented in the literature, but there exists only one study, by Spycher and Pruess, that has compared theoretical molar enthalpy predictions with experimental data 1. In this report, we recommend two different models for enthalpy calculations: the CPA equation of state by $\mathrm{Li}$ and Firoozabadi [2, and the $\mathrm{CO}_{2}$ activity coefficient model by Duan and Sun 3. We show that the CPA equation of state, which has been demonstrated to provide good agreement with density and solubility data, also accurately calculates molar enthalpies of pure $\mathrm{CO}_{2}$, pure water, and both $\mathrm{CO}_{2}$-rich and aqueous $\left(\mathrm{H}_{2} \mathrm{O}\right.$-rich) mixtures of the two species. It is applicable to a wider range of conditions than the Spycher and Pruess model. In aqueous sodium chloride $(\mathrm{NaCl})$ mixtures, we show that Duan and Sun's model yields accurate results for the partial molar enthalpy of $\mathrm{CO}_{2}$. It can be combined with another model for the brine enthalpy to calculate the molar enthalpy of $\mathrm{H}_{2} \mathrm{O}-\mathrm{CO}_{2}-\mathrm{NaCl}$ mixtures. We conclude by explaining how the CPA equation of state may be modified to further improve agreement with experiments. This generalized CPA is the basis of our future work on this topic.
\end{abstract}

\section{Introduction}

Mixtures of carbon dioxide $\left(\mathrm{CO}_{2}\right)$ with water, including brines, are important in a number of industries, such as food products, combustion systems, cosmetics, and petrochemicals. They also play a major role in Earth science applications like geological carbon sequestration, in which $\mathrm{CO}_{2}$ is injected and stored in saline aquifers located deep in the Earth's subsurface 4, 5, 6, 7, 8, 9, 10, In all of these applications, accurate models of thermodynamic properties are essential. The models must

\footnotetext{
*myint1@llnl.gov

${ }^{\dagger}$ hao10llnl.gov

$\ddagger$ abbas.firoozabadi@yale.edu
} 
often be reliable over a wide range of temperatures and pressures. This is the case, for example, in the work of the National Risk Assessment Partnership (NRAP), which is a collaborative effort between several United States Department of Energy national laboratories [11]. One of NRAP's main missions is to predict the risk of $\mathrm{CO}_{2}$ leakage from storage reservoirs (deep saline aquifers) to overlying shallow aquifers, which are potential sources of drinking water. The models employed by NRAP need to compute thermodynamic properties in the range of conditions between those of the shallow aquifers, which are roughly between $290-300 \mathrm{~K}$ and 3-20 bar, and those of the $\mathrm{CO}_{2}$ storage reservoirs, which are between 323-423 K and 100-500 bar, depending on the subsurface depth.

Thermodynamic properties of greatest practical interest can be classified into three categories: volumetric (e.g., density), phase behavior (e.g., solubility), and thermal (e.g., enthalpy). For mixtures with $\mathrm{CO}_{2}$ and water, there are several different models that can be used to compute the density and/or solubility over a wide range of conditions. Enthalpy models, however, are much more rare. This may be a reflection of the fact that enthalpy measurements are far less common than density or solubility measurements. To the best of our knowledge, there has been only one reference that has compared theoretical molar enthalpy (or specific enthalpy) predictions with experimental data [1]. This study by Spycher and Pruess has been limited to pure components and to mixtures of $\mathrm{CO}_{2}$ with pure water, not brines. Mixtures with pure water may be of direct interest to the industrial applications listed above. For carbon sequestration, fresh water is sometimes used as a surrogate for the brine in which $\mathrm{CO}_{2}$ is dissolved. Nevertheless, in some cases it may be desirable to represent the saline environment of the aquifers with true brine properties.

The main goal of this report is to identify models that can accurately calculate the enthalpy of mixtures containing $\mathrm{CO}_{2}$ and water. For mixtures with pure water (not electrolytic solutions), we promote use of the cubic-plus-association (CPA) equation of state (EOS) developed by Li and Firoozabadi [2. The CPA equation of state is applicable to pure components as well as to aqueous mixtures containing substances such as $\mathrm{CO}_{2}$, hydrogen sulfide $\left(\mathrm{H}_{2} \mathrm{~S}\right)$ and hydrocarbons. For these mixtures, it has been demonstrated to provide good agreement with experimental density and solubility data, but no comparisons have been made with enthalpy data. In fact, simultaneous prediction of phase equilibria and enthalpy is considered one of the remaining challenges for CPA equations of state [12]. In fluids where water is not present, such as in hydrocarbon- $\mathrm{CO}_{2}$ mixtures, the CPA reduces to the widely-used Peng-Robinson equation of state. The CPA has been successfully implemented in codes that simulate three-phase (oil, water, gas) flows [13. For aqueous mixtures of $\mathrm{CO}_{2}$ dissolved in brines, we recommend using the Duan and Sun activity coefficient model to compute the partial molar enthalpy of $\mathrm{CO}_{2}[3$. We consider brines composed only of water and sodium chloride $(\mathrm{NaCl})$, which is the predominant salt found in deep saline aquifers.

The report is organized as follows. We present molar enthalpy calculations from the CPA equation of state for pure components and $\mathrm{CO}_{2}-\mathrm{H}_{2} \mathrm{O}$ mixtures in Sections 2 and 3 , respectively. We show that it is applicable to a wider range of conditions than the Spycher and Pruess model [1]. In an effort to make the CPA EOS accessible and readily usable, extensive details- much more than presented in the original research paper [2]- are included in Appendices A and B. Enthalpy calculations of $\mathrm{H}_{2} \mathrm{O}-\mathrm{CO}_{2}-\mathrm{NaCl}$ mixtures using the Duan and Sun model are presented in Section 3.2 and Appendix C. Although CPA accurately predicts molar enthalpies, it exhibits relatively poor agreement with experimental excess enthalpy data. Section 4 together with B.2 B.4 explain how the $\mathrm{Li}$ and Firoozabadi version of the CPA equation of state may be generalized to improve the agreement with excess enthalpy data. This generalized model is the starting point of our future work that we outline in Section 5 


\section{Enthalpy of pure components}

\section{$2.1 \quad \mathrm{CO}_{2}$}

The molar enthalpy $h$ is given by $(30)$, which for a pure component fluid simplifies to

$$
h(T, P)=-\left.R T^{2}\left(\frac{\partial \ln \phi(T, P)}{\partial T}\right)\right|_{P, \mathbf{n}}+h^{\mathrm{ig}} .
$$

The fugacity coefficient $\phi$ of the pure component can be obtained from (85), which is the expression for the fugacity coefficient from $\mathrm{Li}$ and Firoozabadi's version of the CPA EOS. Since $\mathrm{CO}_{2}$ is not selfassociating (hydrogen bonds are unable to form between $\mathrm{CO}_{2}$ molecules), this expression simplifies tremendously because the association contribution to $\ln \phi$, which involves the site fractions $\chi_{i}$, is zero. For these non-self-associating substances, Li and Firoozabadi's CPA EOS is equivalent to the Peng-Robinson EOS. The molar enthalpy $h^{\mathrm{ig}}$ of $\mathrm{CO}_{2}$ in the ideal gas state is found by integrating, with respect to temperature, the correlation for the constant pressure heat capacity presented in Appendix C of the textbook by Smith, Van Ness, and Abbott [14]:

$$
h^{\mathrm{ig}}=R\left(a T+b \frac{T^{2}}{2}-\frac{c}{T}\right),
$$

where $a=5.457, b=1.045 \times 10^{-3} \mathrm{~K}^{-1}$, and $c=-1.157 \times 10^{5} \mathrm{~K}^{2}$. For pure components, we compare theoretical calculations to experimental data from the National Institute of Standards and Technology (NIST) [15]. In order for the calculations to be compatible with the data, the same reference point for the enthalpy must be chosen. NIST presents their enthalpy data using a reference point where the internal energy of liquid water along the vapor-liquid (VLE) equilibrium curve at $273.16 \mathrm{~K}$ is assigned to be zero. With this choice of reference point, NIST reports the molar enthalpy of $\mathrm{CO}_{2}$ at $273.16 \mathrm{~K}$ and 34.861 bar (the $\mathrm{CO}_{2}$ vapor pressure at $273.16 \mathrm{~K}$ ) to be $8.804 \mathrm{~kJ} /$ mole. Therefore, the molar enthalpy at any other temperature $T$ and pressure $P$ is

$$
h(T, P)=-\left.R T^{2}\left(\frac{\partial \ln \phi(T, P)}{\partial T}\right)\right|_{P, \mathbf{n}}+h^{\mathrm{ig}}(T)-h(T=273.16 \mathrm{~K}, P=34.861 \text { bar })+8.804,
$$

where $h(T=273.16 \mathrm{~K}, P=34.861$ bar $)$ is the right-hand side of $(1)$ evaluated at the indicated conditions. Computing the enthalpy in this way circumvents the issue that the reference point for the CPA EOS, which is used to calculate the fugacity coefficient, may not be the same as that for the correlation in $(2)$.

The CPA EOS (or more precisely, the Peng-Robinson EOS) produces accurate $\mathrm{CO}_{2}$ enthalpy predictions over a wide range of temperature and pressure conditions (Figure 1), including those stated in Section 1 for NRAP. The maximum error is less than $0.60 \mathrm{~kJ} / \mathrm{mole}$ in virtually the entire range considered. Although not shown here, we have found that calculations for isotherms above $500 \mathrm{~K}$ follow the trend in Figure 1 of increasingly better agreement with data at higher temperatures. The results are comparable to those from Spycher and Pruess, where the accuracy is stated to be within a few percent [1]. The parameters of the EOS have been fitted to experimental vapor pressure measurements, so that the vapor pressure predictions closely match the data, even near the critical temperature. Except for near the critical temperature, the CPA EOS can also accurately predict the molar enthalpy of vaporization $\Delta h_{\mathrm{vap}}$ [Figure 2(b)], which is defined as the 


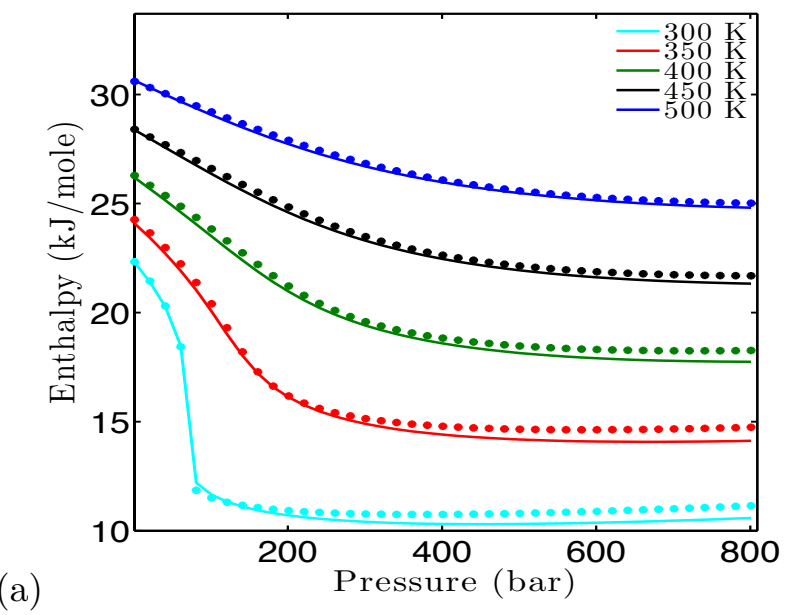

(b)

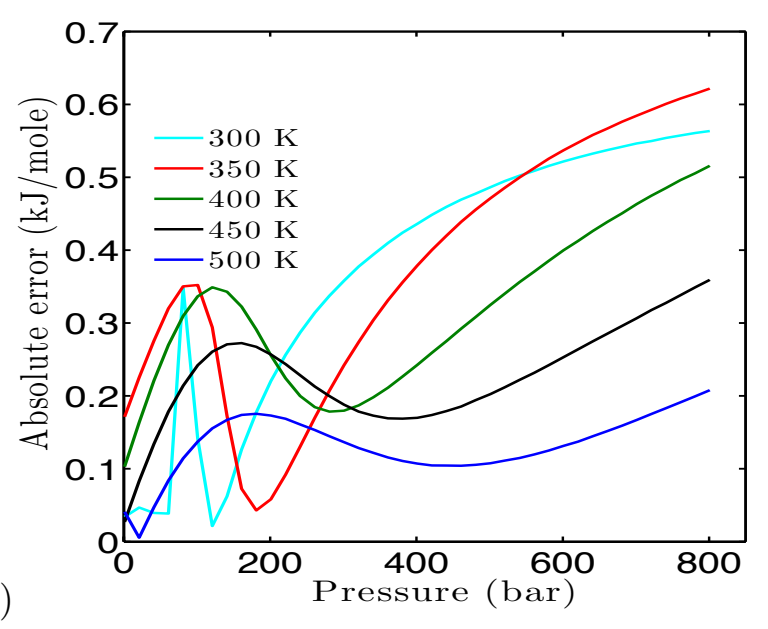

Figure 1: CPA EOS-predicted enthalpies of pure $\mathrm{CO}_{2}$ vs. pressure along five isotherms. Theoretical calculations are depicted in solid lines and are calculated from (3). Experimental data (dots) are obtained from NIST [15]. The absolute error of the predictions in (a) is shown in (b).

difference in the vapor-phase and liquid-phase enthalpies shown in Figure 2(a). The enthalpy of vaporization rapidly approaches zero near the critical point; most of the error near this point comes from the liquid-phase enthalpy predictions. Due to thermodynamic singularities that arise, it is not uncommon for equations of state to have larger errors near the critical point.

\section{$2.2 \quad \mathrm{H}_{2} \mathrm{O}$}

The molar enthalpy of pure water is calculated from (1). In this case, the association contribution to the fugacity coefficient in 85 is non-zero so that the CPA EOS yields different results from the Peng-Robinson EOS on which it is based. The molar enthalpy $h^{\text {ig }}$ of water as an ideal gas can be calculated using (2), with $a=3.470, b=1.450 \times 10^{-3} \mathrm{~K}^{-1}$, and $c=0.121 \times 10^{5} \mathrm{~K}^{2}$ [14]. An alternative and slightly more accurate correlation for $h^{\text {ig }}$ has been presented by Cooper [16]. Using the same reference point mentioned in the previous section (internal energy of liquid water along the VLE curve at $273.16 \mathrm{~K}$ ), we calculate the enthalpy according to

$$
h(T, P)=-\left.R T^{2}\left(\frac{\partial \ln \phi(T, P)}{\partial T}\right)\right|_{P, \mathbf{n}}+h^{\mathrm{ig}}(T)-h(T=273.16 \mathrm{~K}, P=0.006117 \text { bar }),
$$

where 0.006117 bar is the vapor pressure of water at $273.16 \mathrm{~K}$. Equation (4) should also contain a term that represents the difference between the enthalpy and the internal energy of water at ( $T=273.16 \mathrm{~K}, P=0.006117$ bar), but this term is so small that we neglect it.

Results for five isotherms are shown in Figures 3(a) and 3(b), while the molar enthalpy of vaporization is shown in Figure 3(c). Similar to the case for $\mathrm{CO}_{2}$, the the EOS parameters for water (such as $\epsilon$ and $\kappa$ described in Section B.2.3 are fit to vapor pressure data. Predictions of $\Delta h_{\mathrm{vap}}$ are accurate to within a few percent as long as the temperature is not close to the critical temperature. Spycher and Pruess have obtained good agreement with experimental data for pure water, except for the enthalpy of the vapor along certain regions of the VLE curve [1]. A comparison of their model's predictions with those of the CPA EOS along the VLE curve is shown in Figure 4. The accuracy of 


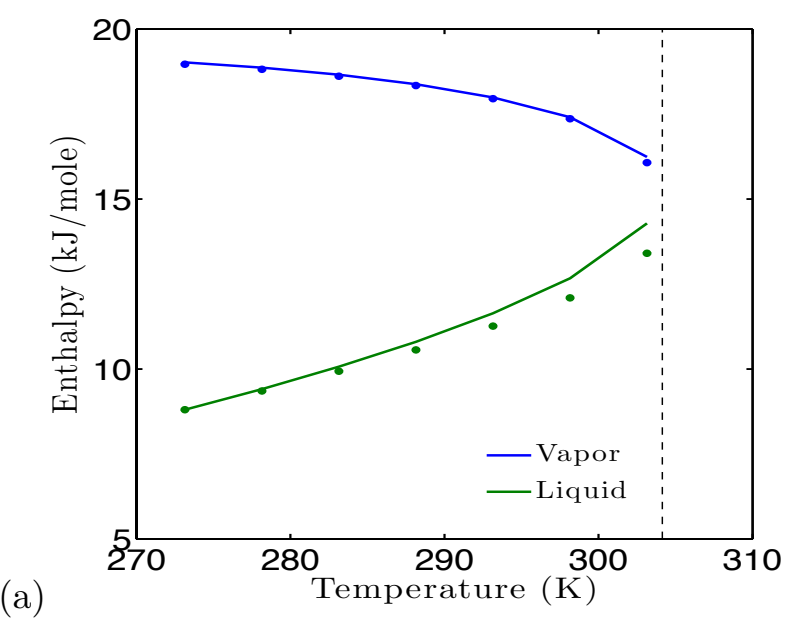

(b)

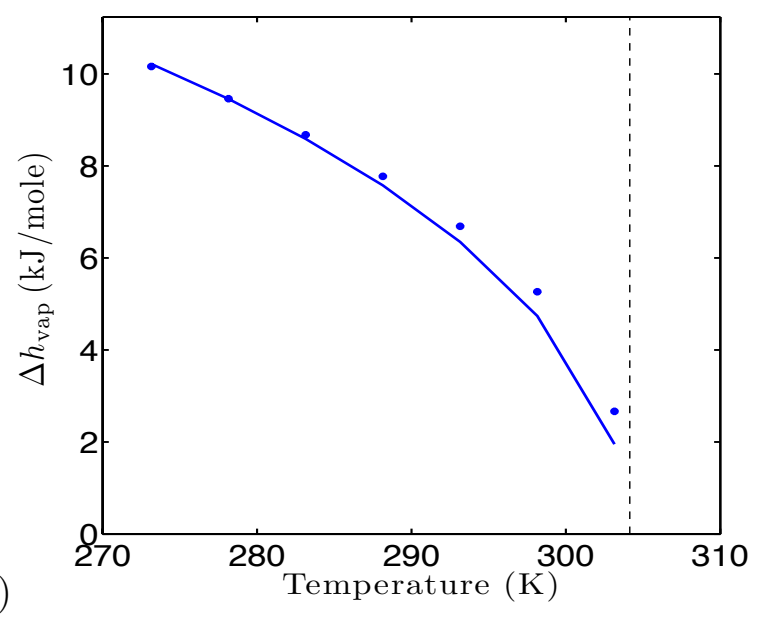

Figure 2: CPA EOS-predicted molar enthalpies of pure $\mathrm{CO}_{2}$ (solid lines) vs. temperature along the vapor-liquid equilibrium (VLE) curve. Experimental data (dots) are obtained from NIST [15]. The dashed vertical line crosses the abscissa at $304.14 \mathrm{~K}$, which is the critical temperature of $\mathrm{CO}_{2}$. (a) Vapor-phase and liquid-phase enthalpies along the VLE curve. (b) presents the molar enthalpy of vaporization $\Delta h_{\mathrm{vap}}$, which is the difference between the vapor and liquid enthalpies in (a).

(a)

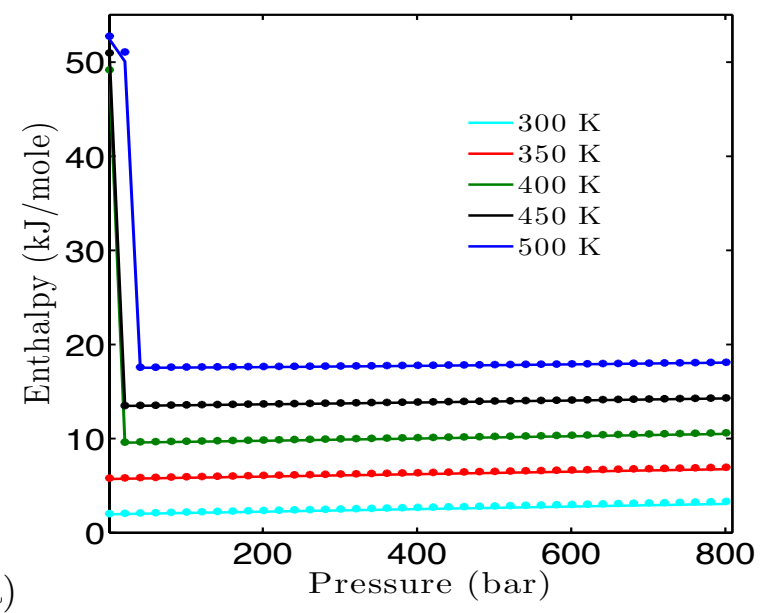

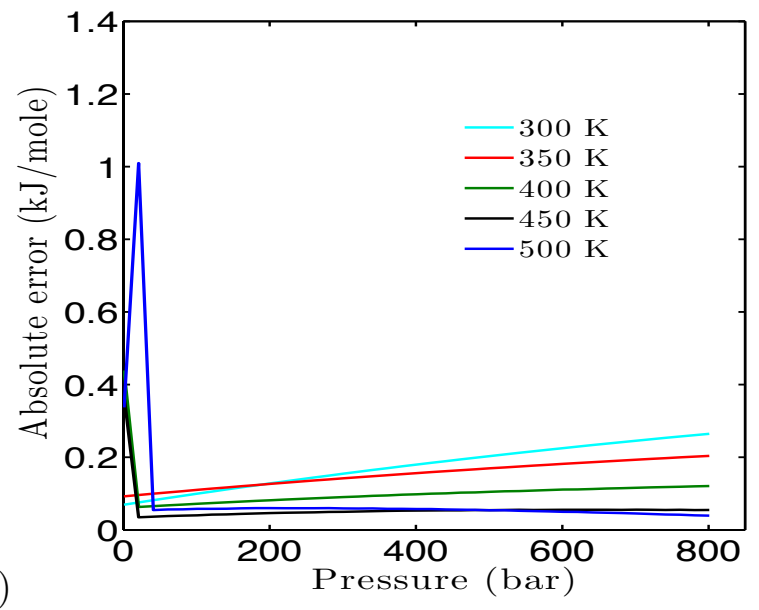

Figure 3: CPA EOS-predicted enthalpies of pure $\mathrm{H}_{2} \mathrm{O}$ vs. pressure along five isotherms. Theoretical calculations are depicted in solid lines and are calculated from (4). Experimental data (dots) are obtained from NIST [15. The absolute error of the predictions in (a) is shown in (b). The $1.0 \mathrm{~kJ} /$ mole error for the $500 \mathrm{~K}$ isotherm is associated with the vapor formed during the phase change at that temperature (see Figure 4). 
the two models is similar in the range of temperatures between $373-550 \mathrm{~K}$ (less than $2.5 \%$ maximum error for both). Above $550 \mathrm{~K}$, the CPA EOS yields noticeably better results, although it fails to reproduce the sharp decrease in the vapor-phase enthalpy near the critical temperature. More importantly, however, Spycher and Pruess state that their model cannot calculate the enthalpy of water below $373 \mathrm{~K}$. This could have practical implications for the applications described in Section 1 . For example, in numerical simulations of $\mathrm{CO}_{2}$ sequestration leakage, Spycher and Pruess' model cannot be used to calculate the enthalpy in the aqueous environments of the shallow aquifers and nearby leakage pathways.

\section{$3 \quad$ Enthalpy of mixtures}

Mixtures of $\mathrm{CO}_{2}$ and water can in general exist as two different phases, a $\mathrm{CO}_{2}$-rich phase and an aqueous $\left(\mathrm{H}_{2} \mathrm{O}\right.$-rich) phase. We treat the $\mathrm{CO}_{2}$-rich phase as being composed only of $\mathrm{CO}_{2}$ and water, while the aqueous phase may have dissolved sodium chloride.

\section{1 $\mathrm{CO}_{2}$-rich phase $\left(\mathrm{CO}_{2}+\mathrm{H}_{2} \mathrm{O}\right)$}

At high $(T, P)$ conditions, the $\mathrm{CO}_{2}$-rich phase may be composed almost entirely of $\mathrm{CO}_{2}$ that exists in a relatively dense supercritical state. The solubility of water in this form of $\mathrm{CO}_{2}$ is less than 2 mole \% [2], and often times it is even much less than that. Since the mixture is almost pure $\mathrm{CO}_{2}$, the Peng-Robinson EOS is a very good approximation to the CPA EOS, and we have already demonstrated in Section 2.1 that it can accurately model the behavior of $\mathrm{CO}_{2}$ over a wide range of $(T, P)$, including supercritical conditions. However, at sufficiently low pressures for a given temperature, $\mathrm{CO}_{2}$ may exist as a gaseous substance. When water vapor is exposed to $\mathrm{CO}_{2}$ at these conditions, the resulting gaseous mixture may have very high concentrations of water, since gases are completely miscible in each other. These mixtures find applications in technologies such as oxy-fuel combustion, which is a method for carbon capture involving the formation of mixtures rich in $\mathrm{CO}_{2}$ and water vapor [17].

Experimental enthalpy data for $\mathrm{CO}_{2}-\mathrm{H}_{2} \mathrm{O}$ mixtures, which are much less common than data for the pure components, have been presented by Patel and Eubank [18]. They have studied $\mathrm{CO}_{2}$-rich gaseous mixtures between $323 \mathrm{~K}$ and $498 \mathrm{~K}$ where the water vapor mole fraction can be as high as $50 \%$. The same set of data is also considered by Spycher and Pruess [1]. Patel and Eubank choose the reference to be the enthalpy of the corresponding ideal gas mixture at $T=273.16 \mathrm{~K}$. With this reference, we calculate the molar enthalpy $h(T, P, \mathbf{z})$ of the real fluid mixture from the CPA EOS according to

$$
h(T, P, \mathbf{z})=-\left.R T^{2} \sum_{i=1}^{2} z_{i}\left(\frac{\partial \ln \phi_{i}(T, P, \mathbf{n})}{\partial T}\right)\right|_{P, \mathbf{n}}+h^{\mathrm{ig}}(T)-h^{\mathrm{ig}}(T=273.16 \mathrm{~K}),
$$

where $h^{\mathrm{ig}}(T)=\sum_{i=1}^{2} z_{i} h_{i}^{\mathrm{ig}}(T), z_{i}$ is the mole fraction of component $i$, and the pure component ideal gas enthalpies $h_{i}^{\mathrm{ig}}(T)$ are calculated as described in Section 2, Enthalpy results for several isotherms of $\mathrm{CO}_{2}-\mathrm{H}_{2} \mathrm{O}$ mixtures at two different compositions are shown in Figure 5. Spycher and Pruess' model is more accurate at low pressures. As the pressure increases, the CPA EOS becomes more accurate. The point at which this switch occurs depends on the temperature and the composition; the CPA EOS tends to be more accurate at higher concentrations of water and at lower temperatures. In Figure 5(a), the maximum error for the CPA EOS is $0.26 \mathrm{~kJ} / \mathrm{mole}$. In 

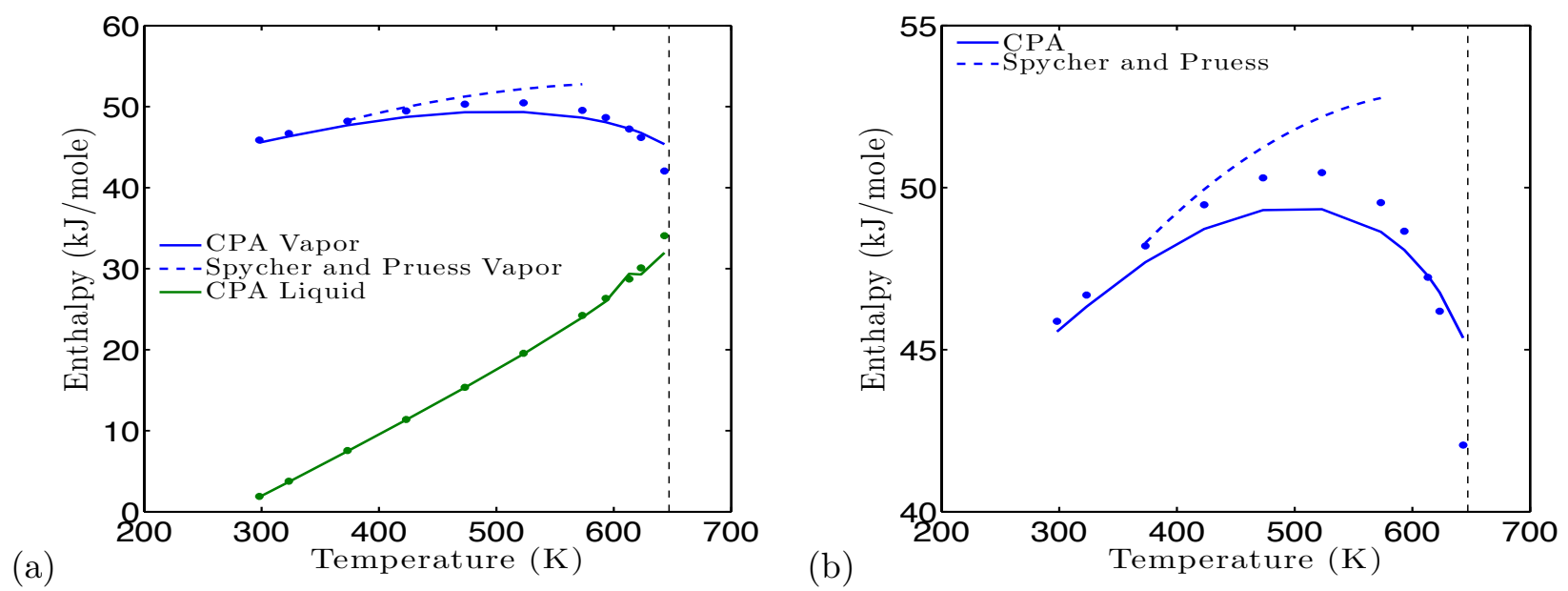

(b)

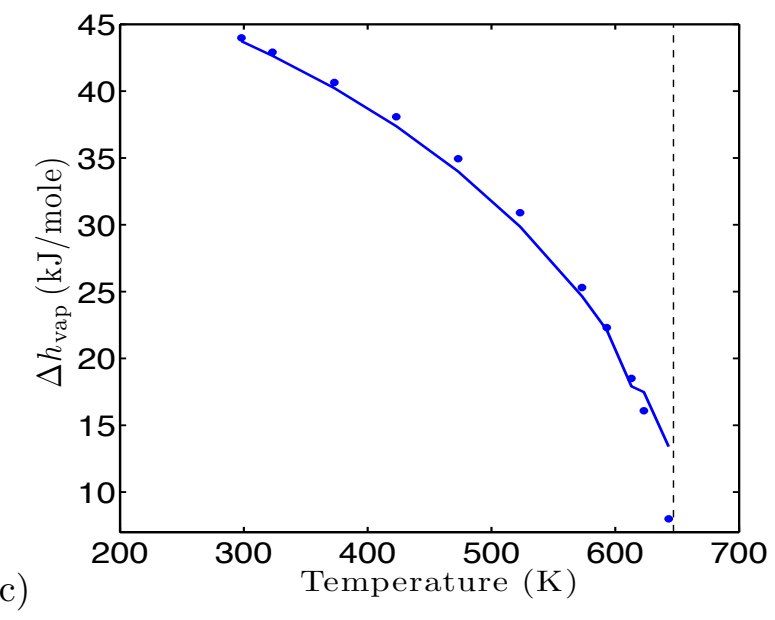

Figure 4: CPA EOS-predicted molar enthalpies of pure $\mathrm{H}_{2} \mathrm{O}$ vs. temperature along the VLE curve. Results from Spycher and Pruess [1] are included for comparison. Experimental data (dots) are obtained from NIST [15]. The dashed line vertical crosses the abscissa at $647.1 \mathrm{~K}$, which is the critical temperature of $\mathrm{H}_{2} \mathrm{O}$. A magnified view of the vapor-phase results in (a) is shown in (b). Predictions of the molar enthalpy of vaporization is compared with experimental data in (c). 
(a)

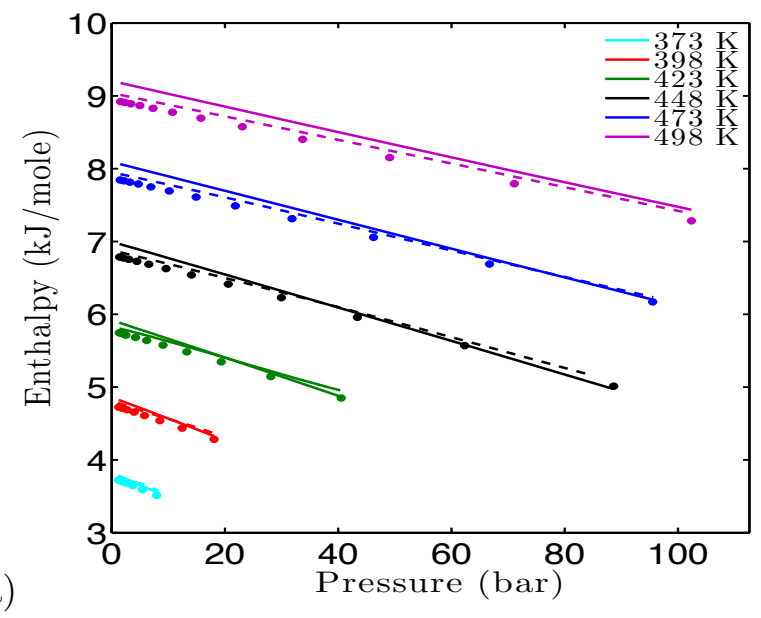

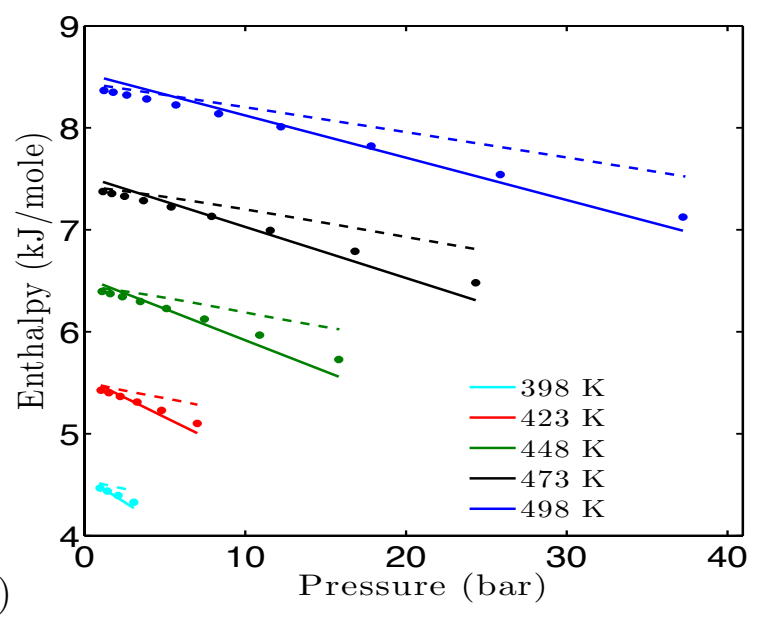

Figure 5: CPA EOS-predicted molar enthalpies of gaseous $\mathrm{CO}_{2}-\mathrm{H}_{2} \mathrm{O}$ mixtures (solid lines) along different isotherms vs. pressure. Experimental data (dots) are obtained from Patel and Eubank [18. Results from Spycher and Pruess (dashed lines) [1] are included for comparison. (a) 9:1 mole ratio of $\mathrm{CO}_{2}$ to $\mathrm{H}_{2} \mathrm{O}$; (b) 1:1 mole ratio of $\mathrm{CO}_{2}$ to $\mathrm{H}_{2} \mathrm{O}$.

Figure 5(b), the maximum error is $0.17 \mathrm{~kJ} /$ mole. Spycher and Pruess state that their maximum error is about $0.40 \mathrm{~kJ} /$ mole $(13 \mathrm{~kJ} / \mathrm{kg})$. Besides the fact that the CPA EOS can simultaneously predict phase equilibria behavior, density, and enthalpy, its main advantage is that it can calculate the enthalpy of pure water or mixtures involving water below $373 \mathrm{~K}$. Although not shown in Figure 5(a), Patel and Eubank present a few enthalpy data points at $348 \mathrm{~K}$ for the $90 \% \mathrm{CO}_{2}$ mixture. The maximum error of the CPA EOS for these points is less than $0.03 \mathrm{~kJ} / \mathrm{mole}$.

\subsection{Aqueous phase $\left(\mathrm{H}_{2} \mathrm{O}+\mathrm{CO}_{2}+\mathrm{NaCl}\right)$}

To the best of our knowledge, there are no reported molar enthalpy measurements for aqueous mixtures with $\mathrm{CO}_{2}$. Measurements of the $\mathrm{CO}_{2}$ molar enthalpy of solution $\Delta h_{\text {sol }}$ are available, however. This quantity is defined as

$$
\Delta h_{\mathrm{sol}}=\bar{H}_{\mathrm{CO}_{2}}\left(T, P, z_{\mathrm{CO}_{2}} \rightarrow 0\right)-h_{\mathrm{CO}_{2}}(T, P) .
$$

That is, $\Delta h_{\text {sol }}$ is the difference between the partial molar enthalpy of $\mathrm{CO}_{2}$ in an infinitely dilute aqueous solvent and the molar enthalpy of pure $\mathrm{CO}_{2}$. Koschel et al. [19] have presented $\Delta h_{\text {sol }}$ data at $323 \mathrm{~K}$ and $373 \mathrm{~K}$ in three different aqueous solvents: pure water, 1 molal $(\mathrm{m}) \mathrm{NaCl}$, and $3 \mathrm{~m} \mathrm{NaCl}$. As explained in Section B.2, the CPA EOS is limited to aqueous-phase mixtures containing only pure water. For such mixtures, Figure 6 compares $\Delta h_{\text {sol }}$ calculations from the CPA EOS with experimental data from Koschel et al. The agreement at $323 \mathrm{~K}$ is poor, while that at $373 \mathrm{~K}$ is relatively good. Since the EOS accurately calculates the molar enthalpy of pure $\mathrm{CO}_{2}$, most of the error in $\Delta h_{\text {sol }}$ comes from calculation of the infinite dilution partial molar enthalpy.

Despite the large error in $\Delta h_{\text {sol }}$, the CPA EOS can still provide accurate predictions of the molar enthalpy $h(T, P, \mathbf{z})$ of the mixture. This can be reasoned as follows. From (26), the molar enthalpy of $\mathrm{H}_{2} \mathrm{O}-\mathrm{CO}_{2}$ mixtures is 

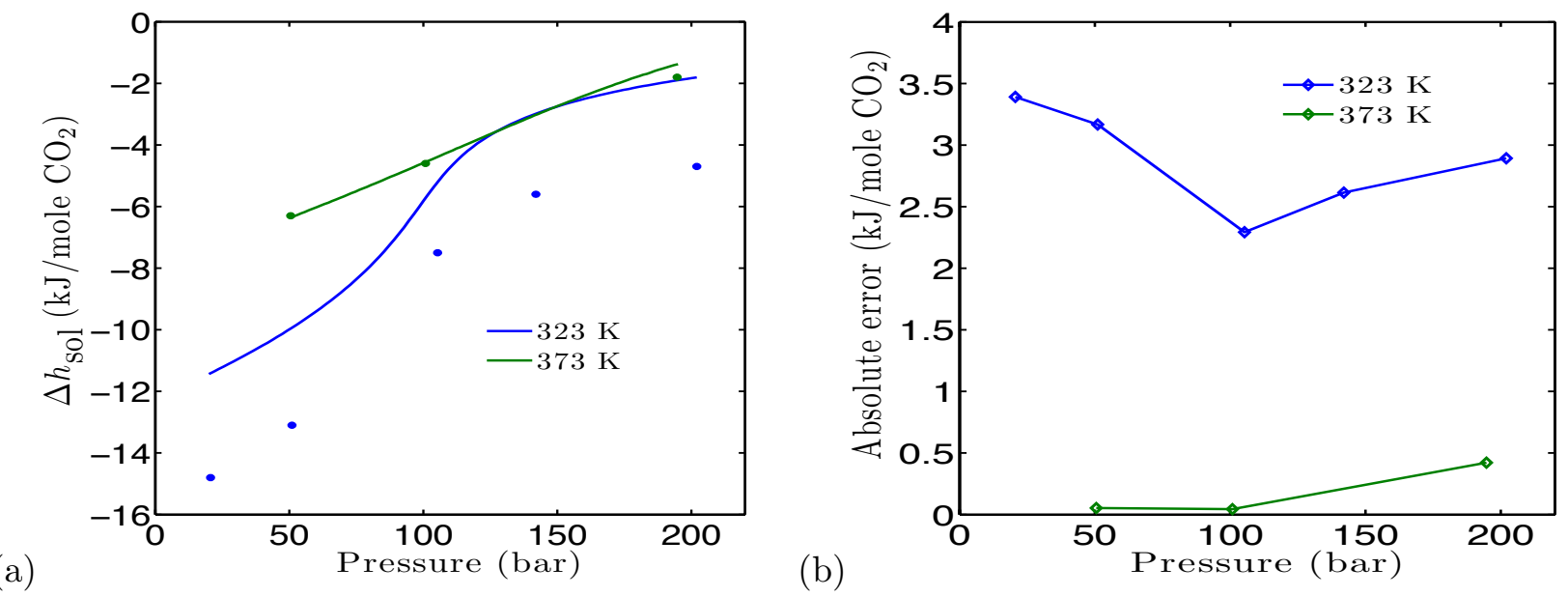

Figure 6: (a) CPA EOS-predicted $\mathrm{CO}_{2}$ molar enthalpy of solution in pure water vs. pressure at two different temperatures. Experimental data are obtained from Koschel et al. [19]. (b) shows the absolute error of the predictions. Despite the large error in $\Delta h_{\text {sol }}$ at $323 \mathrm{~K}$, the EOS can still accurately compute the molar enthalpy $h(T, P, \mathbf{z})$ of $\mathrm{H}_{2} \mathrm{O}-\mathrm{CO}_{2}$ mixtures, as explained in the text.

$$
h(T, P, \mathbf{z})=z_{\mathrm{CO}_{2}} \bar{H}_{\mathrm{CO}_{2}}+z_{\mathrm{H}_{2} \mathrm{O}} \bar{H}_{\mathrm{H}_{2} \mathrm{O}} .
$$

For the temperatures and pressures considered in this report (say, $T<500 \mathrm{~K}$ and $P<500$ bar), the $\mathrm{CO}_{2}$ solubility in pure water is less than 3.5 mole \% [2]. Suppose that the error in the calculation of the partial molar enthalpy $\bar{H}_{\mathrm{CO}_{2}}$ is $4.0 \mathrm{~kJ} / \mathrm{mole} \mathrm{CO}_{2}$, which is a value greater than the maximum error of $3.5 \mathrm{~kJ} / \mathrm{mole} \mathrm{CO}_{2}$ in Figure 6. Then the error in the product $z_{\mathrm{CO}_{2}} \bar{H}_{\mathrm{CO}_{2}}$, assuming that $z_{\mathrm{CO}_{2}}=0.035$, is $0.14 \mathrm{~kJ} /$ mole. The partial molar enthalpy $\bar{H}_{\mathrm{H}_{2} \mathrm{O}}$ of water is similar to the molar enthalpy $h_{\mathrm{H}_{2} \mathrm{O}}$ of the pure component because the aqueous mixture is predominantly water. For example at $323 \mathrm{~K}$, the CPA EOS predicts that the difference between the two quantities is less than $0.006 \mathrm{~kJ} /$ mole $\mathrm{H}_{2} \mathrm{O}$ even for $\mathrm{CO}_{2}$-saturated water at $500 \mathrm{bar}$. It is therefore reasonable to expect the error in $\bar{H}_{\mathrm{H}_{2} \mathrm{O}}$ to be similar to that for $h_{\mathrm{H}_{2} \mathrm{O}}$ shown in Figure 3 , where the maximum error is $0.25 \mathrm{~kJ} /$ mole $\mathrm{H}_{2} \mathrm{O}$ in the liquid phase. Thus, the error in $h(T, P, \mathbf{z})$ over a wide range of conditions will likely be less than $0.40 \mathrm{~kJ} /$ mole $(\approx 0.14 \mathrm{~kJ} / \mathrm{mole}+0.25 \mathrm{~kJ} / \mathrm{mole})$, which is rather small considering the expected magnitude of $h(T, P, \mathbf{z})$. Nonetheless, we propose a generalized version of the Li and Firoozabadi CPA EOS in Section 4, and explain how it may provide better agreement with the excess enthalpy, which is related to $\Delta h_{\text {sol }}$. Another alternative is the Duan and Sun $\mathrm{CO}_{2}$ activity coefficient model [3], which not only agrees better with $\Delta h_{\text {sol }}$ measurements in pure water, but also provides an accurate way to calculate the $\mathrm{CO}_{2}$ partial molar enthalpy (and subsequently $\Delta h_{\text {sol }}$ ) in brine solutions. Their study is the focus of the rest of this section.

Duan and Sun developed their model for the purpose of performing $\mathrm{CO}_{2}$ solubility calculations in pure water and brines. Appendix $\mathrm{C}$ describes how it can also be used to calculate the $\mathrm{CO}_{2}$ partial molar enthalpy $\bar{H}_{\mathrm{CO}_{2}}(T, P, \mathbf{m})$ and $\Delta h_{\text {sol }}$. Here, $\mathbf{m}$ represents the molalities of all the solutes dissolved in the aqueous solvents. Figure 7 compares $\Delta h_{\text {sol }}$ predictions from their model with data from Koschel et al. For mixtures in pure water $(m=0)$, there is marked improvement over the CPA EOS at $323 \mathrm{~K}$. Since $\mathrm{CO}_{2}$ is even less soluble in brines than in pure water, we follow the same reasoning in the preceding paragraph to argue that the $\mathrm{CO}_{2}$ contribution to the error 

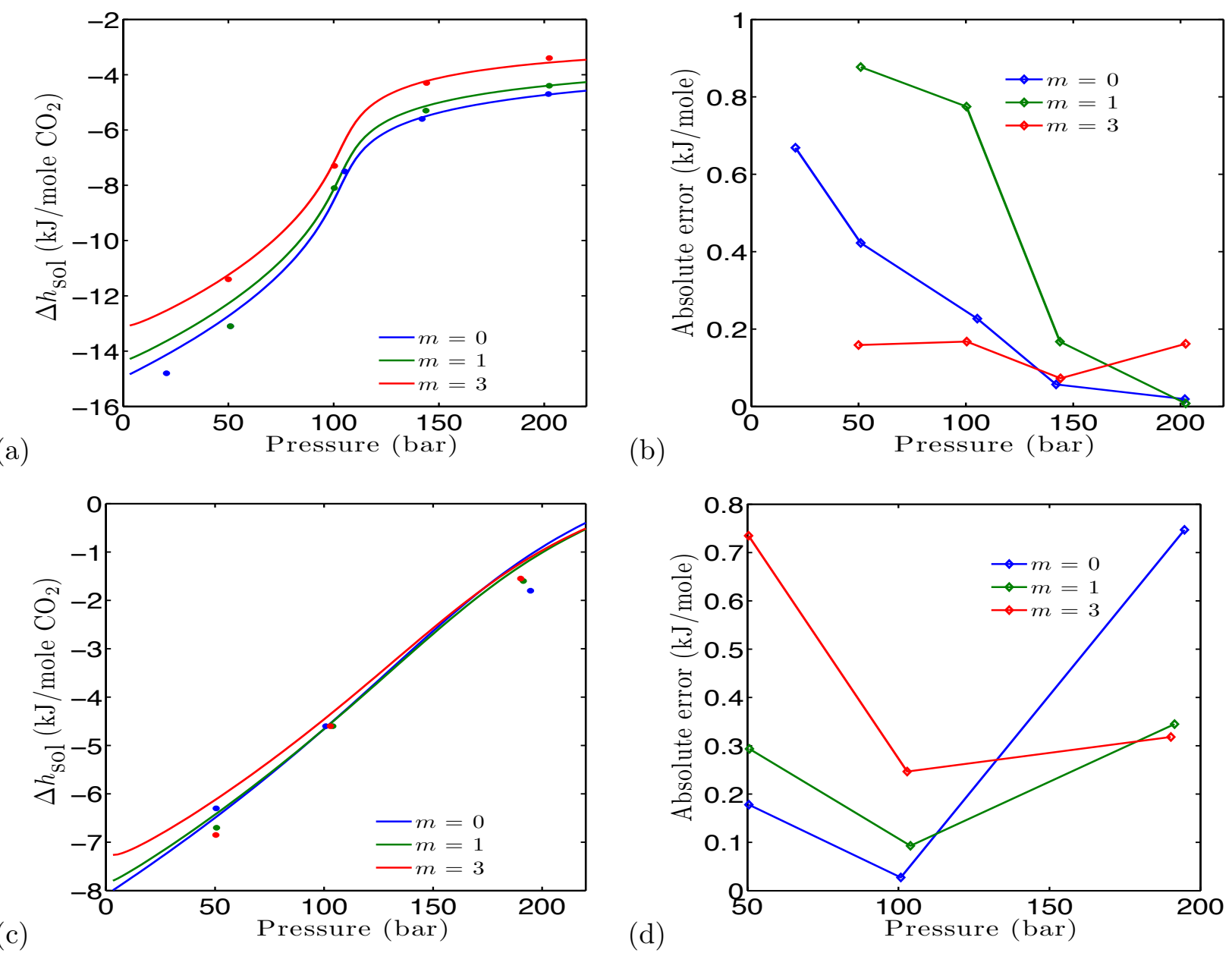

(b)

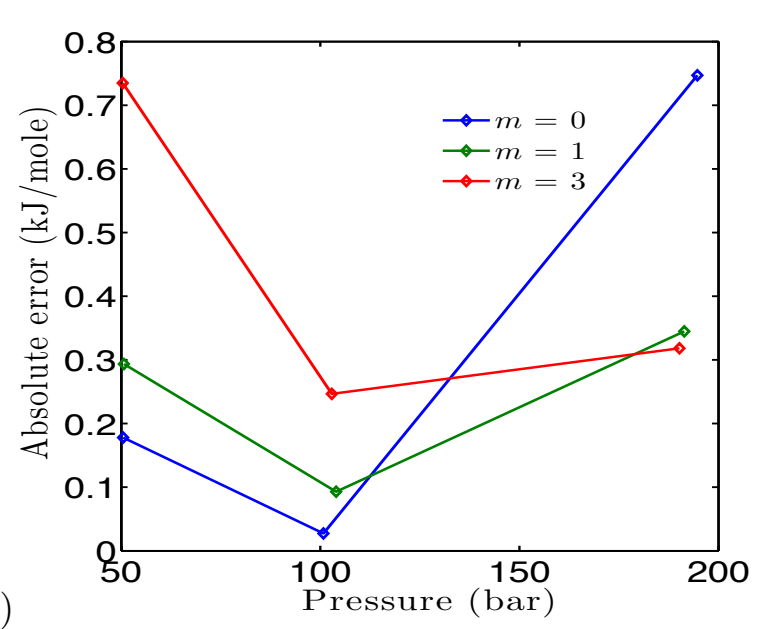

Figure 7: $\mathrm{CO}_{2}$ molar enthalpy of solution derived from Duan and Sun's $\mathrm{CO}_{2}$ activity coefficient model [3] vs. pressure at $323 \mathrm{~K}(\mathrm{a}, \mathrm{b})$ and $373 \mathrm{~K}(\mathrm{c}, \mathrm{d})$ and three different $\mathrm{NaCl}$ molalities $m$. Experimental data are obtained from Koschel et al. [19].

in the molar enthalpy $h(T, P, \mathbf{m})$ of an $\mathrm{H}_{2} \mathrm{O}-\mathrm{CO}_{2}-\mathrm{NaCl}$ aqueous mixture is relatively small as long as $\bar{H}_{\mathrm{CO}_{2}}(T, P, \mathbf{m})$ is predicted to within a few $\mathrm{kJ} /$ mole $\mathrm{CO}_{2}$. The accuracy of Duan and Sun's model is well within this limit, at least for the conditions where experimental data are available (temperatures, pressures, and $\mathrm{NaCl}$ concentrations up to $373 \mathrm{~K}, 200 \mathrm{bar}$, and 3 molal, respectively).

In order to obtain the molar enthalpy $h(T, P, \mathbf{m})$, we must calculate the contributions from water and $\mathrm{NaCl}$. Rather than treating water and $\mathrm{NaCl}$ separately, one approach is to treat them together as a single pseudocomponent, which we label as brine. In this approach, the $\mathrm{H}_{2} \mathrm{O}-\mathrm{CO}_{2}-\mathrm{NaCl}$ mixture may be thought of as a binary brine- $\mathrm{CO}_{2}$ mixture. A correlation for the specific enthalpy $h_{\text {brine }}$ of sodium chloride solutions, in units of $\mathrm{kJ} / \mathrm{kg}$ brine, has been presented by Michaelides [20]. This correlation contains an error in one of the coefficients that was corrected by a later study [21]. For every kilogram of water in the brine- $\mathrm{CO}_{2}$ solution, the total moles are

$$
\frac{1000}{M_{\mathrm{H}_{2} \mathrm{O}}}+m_{\mathrm{NaCl}}+m_{\mathrm{CO}_{2}}
$$


and the total mass of brine $\left(\mathrm{H}_{2} \mathrm{O}+\mathrm{NaCl}\right)$, in units of $\mathrm{kg}$ brine $/ \mathrm{kg} \mathrm{H}_{2} \mathrm{O}$, is

$$
1+m_{\mathrm{NaCl}} \frac{M_{\mathrm{NaCl}}}{1000},
$$

where $M_{\mathrm{NaCl}}=58.44 \mathrm{~g} /$ mole is the molecular weight of the salt. As a result, the enthalpy of the solution per kilogram of water is approximately given by

$$
m_{\mathrm{CO}_{2}} \bar{H}_{\mathrm{CO}_{2}}(T, P, \mathbf{m})+\left(1+m_{\mathrm{NaCl}} \frac{M_{\mathrm{NaCl}}}{1000}\right) h_{\text {brine }} .
$$

The molar enthalpy is therefore

$$
h(T, P, \mathbf{m})=\left[\frac{m_{\mathrm{CO}_{2}}}{\frac{1000}{M_{\mathrm{H}_{2} \mathrm{O}}}+m_{\mathrm{NaCl}}+m_{\mathrm{CO}_{2}}}\right] \bar{H}_{\mathrm{CO}_{2}}(T, P, \mathbf{m})+\left[\frac{\left(1+m_{\mathrm{NaCl}} \frac{M_{\mathrm{NaCl}}}{1000}\right)}{\frac{1000}{M_{\mathrm{H}_{2} \mathrm{O}}}+m_{\mathrm{NaCl}}+m_{\mathrm{CO}_{2}}}\right] h_{\text {brine }} .
$$

Equation (6) is an approximation because $h(T, P, \mathbf{m})$ should involve the specific enthalpy of brine that contains dissolved $\mathrm{CO}_{2}$, and not the specific enthalpy of brine by itself. However, since the mixture is predominantly brine, the two quantities are not expected to be very different, just like how $\bar{H}_{\mathrm{H}_{2} \mathrm{O}}$ and $h_{\mathrm{H}_{2} \mathrm{O}}$ are similar. Michaelides states $h_{\text {brine }}$ is accurate to within $3 \%$. This value is comparable to the error of the liquid water molar enthalpy in Figure 3. Since Duan and Sun's model can compute $\bar{H}_{\mathrm{CO}_{2}}$ more accurately than the CPA EOS for most of the conditions, it is reasonable to expect $h(T, P, \mathbf{m})$ predictions to deviate from experimental data by no more than $0.40 \mathrm{~kJ} / \mathrm{kg}$ (the maximum estimated error for the CPA EOS) over a wide range of conditions.

\section{Excess enthalpy}

This section explains how relaxing two of the assumptions in Li and Firoozabadi's version of the CPA EOS may allow for better agreement with experimental data for the molar excess enthalpy. This quantity is distinct from the molar enthalpy that we have so far considered. From the general definition in (24), the molar excess enthalpy is

$$
h^{\text {excess }}(T, P, \mathbf{z})=h(T, P, \mathbf{z})-h^{\mathrm{im}}(T, P, \mathbf{z}),
$$

where $h^{\mathrm{im}}(T, P, \mathbf{z})$ is the molar enthalpy of the corresponding ideal mixture at the conditions $(T, P, \mathbf{z})$. Since the molar enthalpy of an ideal mixture is calculated from the pure component enthalpies (see Section A.3), $\Delta h_{\text {sol }}$ in (5) is equivalent to the partial molar excess enthalpy of $\mathrm{CO}_{2}$ in an infinitely dilute mixture. Therefore, the generalized CPA EOS proposed in this section is expected to also improve the agreement depicted in Figure 6. Bottini and Saville have measured the molar excess enthalpy of gaseous $\mathrm{CO}_{2}-\mathrm{H}_{2} \mathrm{O}$ mixtures in the temperature range $520-620 \mathrm{~K}$, pressure range $14-45$ bar, and $\mathrm{CO}_{2}$ mole fractions between $20-78 \%$ [22]. For these mixtures, the CPA EOS yields an average absolute error of $1.1 \mathrm{~kJ} / \mathrm{kg}$ (this corresponds to a $13.3 \%$ error) and a maximum error of $5.6 \mathrm{~kJ} / \mathrm{kg}$. Figure 8 compares CPA predictions of the molar excess enthalpy of gaseous $\mathrm{CO}_{2}-\mathrm{H}_{2} \mathrm{O}$ mixtures with data from two other studies. The absolute error can be a significant fraction of the excess enthalpy, especially at the higher temperatures. Nevertheless, these results are an improvement over those from Spycher and Pruess, who state that their average 


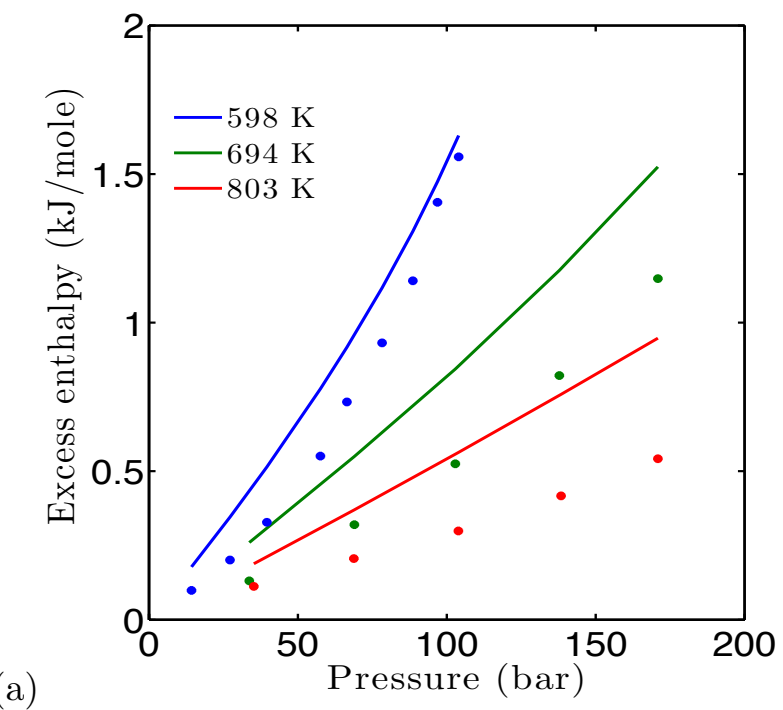

(a)

Figure 8: CPA EOS-predicted molar excess enthalpies of gaseous $\mathrm{CO}_{2}-\mathrm{H}_{2} \mathrm{O}$ mixtures vs. pressure at three different $T$. The experimental data at $598 \mathrm{~K}$ are from [24] and represent 1:1 mole ratio mixtures of $\mathrm{CO}_{2}$ to $\mathrm{H}_{2} \mathrm{O}$. Data at $694 \mathrm{~K}$ and $803 \mathrm{~K}$ are from [25], where the composition ranges between 47-51 mole \% of $\mathrm{CO}_{2}$. (b) shows the absolute error of the results in (a).

absolute error for 1:1 gaseous mixtures is about $0.30 \mathrm{~kJ} / \mathrm{mole}(10 \mathrm{~kJ} / \mathrm{kg})$, and their average error for the Bottini and Saville study is $2.4 \mathrm{~kJ} / \mathrm{kg}$ [1]. We note that an earlier study of $\mathrm{H}_{2} \mathrm{O}-\mathrm{CO}_{2}$ mixtures has achieved good agreement with excess enthalpy data up to $598 \mathrm{~K}$, although their model also exhibits significant errors at higher temperatures [23]. This study uses optimization techniques to fit parameters to many different sets of data. The generalized corresponding states principle is employed to make the parameter space more manageable. Their model is targeted towards only the aqueous phase, however. It is not applicable to pure $\mathrm{CO}_{2}$ or to $\mathrm{CO}_{2}$-rich mixtures with water vapor, like the 90 mole $\% \mathrm{CO}_{2}$ mixtures in Figure 5(a).

We may explain the behavior in Figure 8 by considering the nature of intermolecular forces, particularly hydrogen bonding, that exist in mixtures of $\mathrm{CO}_{2}$ and $\mathrm{H}_{2} \mathrm{O}$ (see Sections B.1 and B.2). Breaking hydrogen bonds requires an energy input on the order of $10 \mathrm{~kJ} /$ mole [26], which is at least an order of magnitude larger than the excess enthalpy values. The large disparity suggests that the excess enthalpies are rather small in magnitude, so that even small discrepancies between theory and experiment appear as large relative errors in Figure 8 (b). The discrepancy may be largely due to the two assumptions listed in Section B.5. The assumptions state that the $\alpha$ (hydrogen-bond donor) and $\beta$ (hydrogen-bond acceptor) sites are equally numerous on a molecule of component $i$ and associate symmetrically so that their site fractions $\chi_{i \alpha}$ and $\chi_{i \beta}$ can be described by a single function $\chi_{i}$. In order to see the consequences of these assumptions, let us examine, as a representative example, a 1:1 $\mathrm{CO}_{2}-\mathrm{H}_{2} \mathrm{O}$ gaseous mixture at $598 \mathrm{~K}$ and 66.5 bar. The relevant values are:

- Experimental molar excess enthalpy $=0.73 \mathrm{~kJ} / \mathrm{mole}$.

- CPA-predicted molar excess enthalpy $=h(T, P, \mathbf{z})-h^{\mathrm{im}}(T, P, \mathbf{z})=0.92 \mathrm{~kJ} / \mathrm{mole}$.

- CPA-predicted molar enthalpy departure function $=h(T, P, \mathbf{z})-h^{\mathrm{ig}}(T, P, \mathbf{z})=-1.46 \mathrm{~kJ} / \mathrm{mole}$. 
Qualitatively, the signs and relative magnitudes of the excess enthalpy and departure function are as expected. The departure function should be negative because the intermolecular interactions present in the real (non-ideal) mixture reduce the energy of the mixture compared to the noninteracting ideal gas mixture. The reason why the excess enthalpy is positive can be understood by considering the process of mixing $\mathrm{CO}_{2}$ with water. Mixing involves three processes:

1. Breaking $\mathrm{CO}_{2}-\mathrm{CO}_{2}$ intermolecular forces, primarily London dispersion forces.

2. Breaking $\mathrm{H}_{2} \mathrm{O}-\mathrm{H}_{2} \mathrm{O}$ intermolecular forces, the most prominent being hydrogen bonds.

3. Forming $\mathrm{CO}_{2}-\mathrm{H}_{2} \mathrm{O}$ intermolecular forces, the most prominent being hydrogen bonds.

The first two require energy input while the last one releases energy. The $\mathrm{CO}_{2}-\mathrm{H}_{2} \mathrm{O}$ hydrogen bonds are not taken into account in the ideal mixture, since the ideal mixture enthalpy is calculated from the pure component enthalpies. These hydrogen bonds are weaker than $\mathrm{H}_{2} \mathrm{O}-\mathrm{H}_{2} \mathrm{O}$ hydrogen bonds because $\mathrm{C}$ is more electronegative than $\mathrm{H}$, so the lone pairs of electrons on the oxygens in $\mathrm{CO}_{2}$ are less effective hydrogen-bond acceptors than the lone pairs on the oxygen in $\mathrm{H}_{2} \mathrm{O}$. As a result, the real mixture should be higher in energy than the ideal mixture, meaning a positive excess enthalpy.

A molecule of $\mathrm{CO}_{2}$ has four $\beta$ sites, representing the 4 lone pairs on the two oxygens, and no $\alpha$ sites (hydrogens). Treating the $\alpha$ and $\beta$ sites as symmetric and equally numerous per molecule will overpredict the number of $\mathrm{CO}_{2}-\mathrm{H}_{2} \mathrm{O}$ hydrogen bonds formed since $\mathrm{CO}_{2}$ does not have any $\alpha$ sites. Consequently, overprediction of the number of $\mathrm{CO}_{2}-\mathrm{H}_{2} \mathrm{O}$ hydrogen bonds will lead to an underprediction of the number of $\mathrm{H}_{2} \mathrm{O}-\mathrm{H}_{2} \mathrm{O}$ hydrogen bonds in the mixture. This is because if a $\mathrm{H}_{2} \mathrm{O}$ molecule forms a hydrogen bond with $\mathrm{CO}_{2}$, it has fewer sites available to form a hydrogen bond with another $\mathrm{H}_{2} \mathrm{O}$. As a result, the theoretically-predicted excess enthalpy will be more positive than the experimental value, as we have observed. The small magnitude of the excess enthalpy is also consistent with our discussion above. We have reasoned that $h^{\text {excess }}$ is positive because $\mathrm{CO}_{2}-\mathrm{H}_{2} \mathrm{O}$ hydrogen bonds are weaker than $\mathrm{H}_{2} \mathrm{O}-\mathrm{H}_{2} \mathrm{O}$ hydrogen bonds. This is due to the fact that carbon is more electronegative than hydrogen. However, since carbon is only slightly more electronegative than hydrogen, we would expect the excess enthalpy to be quite small, certainly much less than the energy required to break a hydrogen bond, which is $\mathcal{O}(10 \mathrm{~kJ} / \mathrm{mole})$.

In order to achieve better agreement with excess enthalpy measurements, Li and Firoozabadi's CPA EOS must be modified so that it distinguishes between $\alpha$ and $\beta$ sites, treating them asymmetrically. We suggest relaxing the two assumptions in Section B.5 so that equations in Sections B.3 and B.4 for the fugacity coefficients $\phi_{i}$ (on which all EOS predictions of the density, solubility, and enthalpy are based), pressure, and compressibility factor $Z$ are solved instead. These equations are derived from the departure function in (60) or equivalently, the one in (51). From a practical perspective, the asymmetric treatment will introduce additional cross-association parameters (see Section B.2.3 that can be fit to excess enthalpy data. The pure component parameters that exist in the current model, such as $\epsilon$ and $\kappa$ described in Section B.2.3, do not need to be modified.

\section{Conclusions and future work}

We have shown that the CPA EOS accurately calculates the molar enthalpy of pure $\mathrm{CO}_{2}$, pure water, $\mathrm{CO}_{2}$-rich mixtures, and aqueous mixtures for the conditions relevant to the applications discussed in Section 11. Since the CPA EOS has previously been demonstrated to provide good 
agreement with density and solubility data [2], it can therefore serve as a single, unified model for thermodynamic property predictions of all of these fluids. Compared to the Spycher and Pruess model [1], the CPA EOS more accurately calculates the vapor-phase enthalpies of pure water at temperatures above $550 \mathrm{~K}$ along the vapor-liquid equilibrium curve (Figure 4). For $\mathrm{CO}_{2}-\mathrm{H}_{2} \mathrm{O}$ mixtures, the CPA is more accurate at lower temperatures, higher pressures, and higher water concentrations (although both models predict the molar enthalpy data in Figure 5 well). For practical purposes, the most important difference between the two models is that the CPA EOS is not limited only to temperatures above $373 \mathrm{~K}$ for pure water or water-containing mixtures.

The CPA EOS is not applicable to aqueous $\mathrm{H}_{2} \mathrm{O}-\mathrm{CO}_{2}-\mathrm{NaCl}$ mixtures, however. For these brine mixtures, we suggest calculating the molar enthalpy with (6). We have argued that (6) is expected to be accurate to within $0.40 \mathrm{~kJ} / \mathrm{mole}$, at least for the conditions where enthalpy data are available [19] (temperatures, pressures, and $\mathrm{NaCl}$ concentrations up to $373 \mathrm{~K}, 200$ bar, and 3 molal, respectively). Equation (6) involves the partial molar enthalpy of $\mathrm{CO}_{2}$, which we obtain by evaluating temperature derivatives of Duan and Sun's $\mathrm{CO}_{2}$ activity coefficient model [3]. In a similar fashion, one can obtain the partial molar volume of $\mathrm{CO}_{2}$ by evaluating pressure derivatives. The density of the brine can then be calculated from this partial molar volume. However, we find that the $\mathrm{CO}_{2}$ partial molar volume derived in this way does not closely agree with experimental data [27]. Density calculations require finer precision than enthalpy calculations because the density increase from $\mathrm{CO}_{2}$ dissolution into water or brine is small. It is less than $2 \%$ even at pressures where the $\mathrm{CO}_{2}$ solubility is high (3 mole \%). As a result, we recommend the density of $\mathrm{H}_{2} \mathrm{O}-\mathrm{CO}_{2}-\mathrm{NaCl}$ mixtures be calculated using the models presented in more recent studies [28, 29].

The CPA EOS may be combined with Duan and Sun's model to form a $\gamma$ - $\phi$ model for $\mathrm{H}_{2} \mathrm{O}-$ $\mathrm{CO}_{2}-\mathrm{NaCl}$ mixtures. In this approach, the CPA EOS is used to compute the density, enthalpy, and composition of the $\mathrm{CO}_{2}$-rich phase, which we treat as being composed only of $\mathrm{CO}_{2}$ and water. For the aqueous phase, Duan and Sun's model is used to calculate the $\mathrm{CO}_{2}$ solubility and the $\mathrm{CO}_{2}$ partial molar enthalpy. The partial molar enthalpy may be combined with the specific enthalpy of brine (computed from the studies [20, 21] described in Section 3.2) to obtain the molar enthalpy (6) of the mixture as whole. The density of the aqueous phase may be calculated from one of the aforementioned models [28, 29]. A more theoretically robust alternative to a $\gamma-\phi$ model is a $\phi-\phi$ model, in which the same EOS is used for both phases. In our future work, we plan to develop a $\phi-\phi$ model by modifying the CPA EOS so that it can accurately predict the thermodynamic properties of both phases. The first step is to generalize the CPA EOS as described in Section 4 to further improve the agreement with $\mathrm{CO}_{2}-\mathrm{H}_{2} \mathrm{O}$ mixture enthalpy data. After this step, we may extend the applicability of the CPA EOS to $\mathrm{H}_{2} \mathrm{O}-\mathrm{CO}_{2}-\mathrm{NaCl}$ mixtures by adding more terms to the departure function 32 to account for intermolecular forces such as ion-ion and ion-dipole interactions. This work will involve many challenges, some of which arise due to the lack of experimental molar enthalpy data for aqueous mixtures. Nonetheless, a systematic and rigorous approach that builds on previous studies of electrolytic solutions [30, 31, 32] may prove to be successful.

\section{Acknowledgments}

This work was performed under the auspices of the U.S. Department of Energy by Lawrence Livermore National Laboratory under Contract DE-AC52-07NA27344. We gratefully acknowledge financial support from Lawrence Livermore National Laboratory and the member institutions of the Reservoir Engineering Research Institute. 


\section{A Basic concepts in the thermodynamics of fluid mixtures}

\section{A.1 Chemical potential, fugacity, fugacity coefficient}

Many fundamental thermodynamic relations for real fluid mixtures are inspired by the corresponding relations for ideal gas mixtures. One such example is the relation between the chemical potential $\mu_{i}^{\text {ig }}(T, P, \mathbf{n})$ of component $i$ in an ideal gas mixture and the pure component ideal gas chemical potential $\mu_{i}^{\mathrm{ig}}(T, P)$. Here, $T$ is the temperature, $P$ is the pressure, $\mathbf{n}$ represents the mole numbers of the mixture's components. The difference between $\mu_{i}^{\mathrm{ig}}(T, P, \mathbf{n})$ and $\mu_{i}^{\mathrm{ig}}(T, P)$ is

$$
\mu_{i}^{\mathrm{ig}}(T, P, \mathbf{n})-\mu_{i}^{\mathrm{ig}}(T, P)=R T \ln z_{i},
$$

where $R$ is the gas constant, and $z_{i}=n_{i} / N$ is the mole fraction of component $i$. Equation (7) can be derived formally using statistical thermodynamics, but it can also be derived in a more informal manner as follows [33]. Suppose a pure component exists as an ideal gas at pressure $P$. If this pure component is mixed isobarically with other ideal gases to form a mixture whose pressure is also $P$, the pressure of the component in the mixture becomes the partial pressure $z_{i} P$ so that

$$
\mu_{i}^{\mathrm{ig}}(T, P, \mathbf{n})-\mu_{i}^{\mathrm{ig}}(T, P)=\left.\int_{P}^{z_{i} P}\left(\frac{\partial \mu_{i}^{\mathrm{ig}}}{\partial P^{\prime}}\right)\right|_{T, \mathbf{n}} \mathrm{d} P^{\prime} .
$$

In our study, we consider only systems where external fields like gravity are negligible and there is only pressure-volume work. For such systems,

$$
\mathrm{d} G=-S \mathrm{~d} T+V \mathrm{~d} P+\sum_{i=1}^{c} \mu_{i} \mathrm{~d} n_{i},
$$

where $G$ is the Gibbs energy, $S$ is the entropy, $V$ is the volume, and $c$ is the number of components. Applying the equality of mixed partial derivatives to (8), we have

$$
\left.\left(\frac{\partial V}{\partial n_{i}}\right)\right|_{T, P, \mathbf{n}_{i}}=\left.\left(\frac{\partial \mu_{i}}{\partial P}\right)\right|_{T, \mathbf{n}},
$$

where the subscript $\mathbf{n}_{i}$ in the derivative of $V$ means that all mole numbers besides $n_{i}$ are held fixed. Using (9) and the volume $V^{\mathrm{ig}}=N R T / P$ of an ideal gas, we obtain

$$
\left.\int_{P}^{z_{i} P}\left(\frac{\partial \mu_{i}^{\mathrm{ig}}}{\partial P^{\prime}}\right)\right|_{T, \mathbf{n}} \mathrm{d} P^{\prime}=\left.\int_{P}^{z_{i} P}\left(\frac{\partial V^{\mathrm{ig}}}{\partial n_{i}}\right)\right|_{T, P^{\prime}, \mathbf{n}_{i}} \mathrm{~d} P^{\prime}=R T \int_{P}^{z_{i} P} \frac{\mathrm{d} P^{\prime}}{P^{\prime}}=R T \ln z_{i},
$$

which leads to (7). Similarly, the chemical potential $\mu_{i}^{\mathrm{ig}}(T, P, \mathbf{n})$ is related to $\mu_{i}^{\mathrm{ig}}\left(T, P^{\prime}, \mathbf{n}\right)$ at a different pressure $P^{\prime}$ (but same temperature and composition) by

$$
\mu_{i}^{\mathrm{ig}}(T, P, \mathbf{n})-\mu_{i}^{\mathrm{ig}}\left(T, P^{\prime}, \mathbf{n}\right)=\left.\int_{P^{\prime}}^{P}\left(\frac{\partial \mu_{i}^{\mathrm{ig}}}{\partial P^{\prime \prime}}\right)\right|_{T, \mathbf{n}} \mathrm{d} P^{\prime \prime}=\left.\int_{P^{\prime}}^{P}\left(\frac{\partial V^{\mathrm{ig}}}{\partial n_{i}}\right)\right|_{T, P^{\prime \prime}, \mathbf{n}_{i}} \mathrm{~d} P^{\prime \prime}=R T \ln \frac{P}{P^{\prime}} .
$$

Combining (7) and (10), the difference between $\mu_{i}^{\mathrm{ig}}(T, P, \mathbf{n})$ and $\mu_{i}^{\mathrm{ig}}\left(T, P^{\prime}, \mathbf{n}^{\prime}\right)$, which is at a different pressure and composition but same temperature, is

$$
\mu_{i}^{\mathrm{ig}}(T, P, \mathbf{n})-\mu_{i}^{\mathrm{ig}}\left(T, P^{\prime}, \mathbf{n}^{\prime}\right)=\left[\mu_{i}^{\mathrm{ig}}(T, P)-\mu_{i}^{\mathrm{ig}}\left(T, P^{\prime}\right)\right]+R T \ln \frac{z_{i}}{z_{i}^{\prime}}=R T \ln \frac{z_{i} P}{z_{i}^{\prime} P^{\prime}}
$$


In analogy to (11), for any fluid (not just ideal gases) each component $i$ is assigned a fugacity $f_{i}=f_{i}(T, P, \mathbf{n})$ so that the difference between $\mu_{i}(T, P, \mathbf{n})$ and $\mu_{i}\left(T, P^{\prime}, \mathbf{n}^{\prime}\right)$ is

$$
\mu_{i}(T, P, \mathbf{n})-\mu_{i}\left(T, P^{\prime}, \mathbf{n}^{\prime}\right)=R T \ln \frac{f_{i}(T, P, \mathbf{n})}{f_{i}\left(T, P^{\prime}, \mathbf{n}^{\prime}\right)} .
$$

In order for (12) to be consistent with (11), the fugacity of component $i$ in an ideal gas mixture must be equal to its partial pressure

$$
f_{i}^{\mathrm{ig}}(T, P, \mathbf{n})=z_{i} P .
$$

The difference in the chemical potential of component $i$ in a real fluid and the chemical potential of that same component in an ideal gas mixture at the same $(T, P, \mathbf{n})$ is

$$
\mu_{i}(T, P, \mathbf{n})-\mu_{i}^{\mathrm{ig}}(T, P, \mathbf{n})=R T \ln \left(\frac{f_{i}}{z_{i} P}\right)=R T \ln \phi_{i}
$$

where

$$
\phi_{i}(T, P, \mathbf{n})=\frac{f_{i}(T, P, \mathbf{n})}{z_{i} P},
$$

is the fugacity coefficient of component $i$. Since any fluid mixture behaves like its corresponding ideal gas mixture as the pressure $P$ decreases to zero, $f_{i}$ becomes $f_{i}^{\mathrm{ig}}(T, P, \mathbf{n})=z_{i} P$ and $\phi_{i}$ becomes unity in this limit:

$$
\begin{gathered}
\lim _{P \rightarrow 0} f_{i}(T, P, \mathbf{n})=z_{i} P, \\
\lim _{P \rightarrow 0} \phi_{i}(T, P, \mathbf{n})=1 .
\end{gathered}
$$

If the fluid in question is a pure component fluid,

$$
\begin{aligned}
& \lim _{P \rightarrow 0} f(T, P)=P, \\
& \lim _{P \rightarrow 0} \phi(T, P)=1 .
\end{aligned}
$$

For a multiphase system of $p$ phases to be in equilibrium, a fundamental thermodynamic requirement [34] is the equality of chemical potentials

$$
\mu_{i}^{1}=\mu_{i}^{2}=\cdots=\mu_{i}^{p} \quad \text { for all } i .
$$

Using (12), the condition for phase equilibrium in (16) can be equivalently stated as the equality of fugacities

$$
f_{i}^{1}=f_{i}^{2}=\cdots=f_{i}^{p} \text { for all } i .
$$

The fugacity $f_{i}$ can be easily calculated from the fugacity coefficient $\phi_{i}$ and vice-versa using (15). If we know how the fugacity of each component varies as a function of temperature, pressure, and composition, (17) can be solved to determine the equilibrium composition (i.e., the solubility of each component) in each phase. Furthermore, we will see in Section (A.3 that other thermodynamic functions, like the density and the enthalpy, can in principle be derived from the fugacities or the 
fugacity coefficients. In essence, the purpose of an equation of state is to provide a relation from which we can derive the functional form of the fugacities for all components. Consequently, the equation of state can be used to derive other thermodynamic functions of the mixture as well. In this sense, the equation of state provides a complete thermodynamic specification of the fluids to which it is applicable.

\section{A.2 Activity coefficient, activity, excess property, partial molar property}

In analogy to (7), an ideal mixture (also called an ideal solution) is defined to be one in which

$$
\mu_{i}^{\mathrm{im}}(T, P, \mathbf{n})-\mu_{i}(T, P)=R T \ln z_{i} .
$$

Here, $\mu_{i}(T, P)$ is the chemical potential of pure component $i$ in the real fluid state, as opposed to the hypothetical ideal gas state in (7). From $(12)$ and $(18)$, the fugacity $f_{i}^{\text {im }}(T, P, \mathbf{n})$ in an ideal mixture and the fugacity $f_{i}(T, P)$ of the pure component are related by

$$
f_{i}^{\mathrm{im}}(T, P, \mathbf{n})=z_{i} f_{i}(T, P) .
$$

An ideal gas mixture can be thought of as an ideal mixture where the pure component fugacity $f_{i}(T, P)=P$ for all conditions such that the fugacity $f_{i}^{\text {im }}(T, P, \mathbf{n})$ in the mixture equals the partial pressure $z_{i} P$. In other words, an ideal gas mixture is an ideal mixture where (13) is satisfied. Equation $(19)$ is a simple relation between a mixture property $f_{i}^{\mathrm{im}}(T, P, \mathbf{n})$ and a pure component property $f_{i}(T, P)$; it is a key feature of ideal mixtures. A similar relation can be formed for a real (non-ideal) fluid mixture by introducing an activity coefficient $\gamma_{i}=\gamma_{i}(T, P, \mathbf{n})$ defined such that

$$
f_{i}(T, P, \mathbf{n})=z_{i} \gamma_{i}(T, P, \mathbf{n}) f_{i}(T, P),
$$

where

$$
\lim _{z_{i} \rightarrow 1} \gamma_{i}=1 .
$$

The activity $\mathrm{a}_{i}$ of component $i$ is defined as

$$
\mathrm{a}_{i}=\gamma_{i} z_{i}=\frac{f_{i}(T, P, \mathbf{n})}{f_{i}(T, P)}
$$

so that for any fluid,

$$
\mu_{i}(T, P, \mathbf{n})-\mu_{i}(T, P)=R T \ln \mathrm{a}_{i} .
$$

With these definitions, one can view an ideal mixture as a real fluid in which $\gamma_{i}=1$ (or equivalently, $\mathrm{a}_{i}=z_{i}$ ) for all components over all conditions. An excess property is defined as

$$
E^{\text {excess }}(T, P, \mathbf{n})=E(T, P, \mathbf{n})-E^{\text {im }}(T, P, \mathbf{n}),
$$

where $E$ is an extensive property (e.g., Gibbs energy) of the mixture.

A related concept to the excess property is the partial molar property. The partial molar property of $E$ with respect to component $i$ is denoted as $\bar{E}_{i}$, and is defined as

$$
\bar{E}_{i}=\left.\left(\frac{\partial E}{\partial n_{i}}\right)\right|_{T, P, \mathbf{n}_{i}} .
$$


One example that we have seen previously in $(9)$ is the partial molar volume

$$
\bar{V}_{i}=\left.\left(\frac{\partial V}{\partial n_{i}}\right)\right|_{T, P, \mathbf{n}_{i}} .
$$

Another example is the chemical potential in (8), which is the partial molar Gibbs energy

$$
\mu_{i}=\bar{G}_{i}=\left.\left(\frac{\partial G}{\partial n_{i}}\right)\right|_{T, P, \mathbf{n}_{i}}
$$

There exists a very important relation between $E$ and its partial molar properties $\bar{E}_{i}$. It can be shown [33, 35, 36] that

$$
E(T, P, \mathbf{n})=\sum_{i=1}^{c} n_{i} \bar{E}_{i},
$$

or on a molar basis, if $e(T, P, \mathbf{z})=E(T, P, \mathbf{n}) / N$,

$$
e(T, P, \mathbf{z})=\sum_{i=1}^{c} z_{i} \bar{E}_{i} .
$$

We denote the set of all mole fractions as $\mathbf{z}=\mathbf{n} / N$. The molar Gibbs energy is

$$
g(T, P, \mathbf{z})=\sum_{i=1}^{c} z_{i} \bar{G}_{i}=\sum_{i=1}^{c} z_{i} \mu_{i} .
$$

Due to complexities arising from intermolecular interactions, the partial molar property of a mixture is in general not equal to the molar property $e_{i}(T, P)=E_{i}(T, P) / n_{i}$ of pure component $i$. That is, $\bar{E}_{i} \neq e_{i}(T, P)$ except in special cases. One such special case is the partial molar enthalpy of an ideal mixture. Section A.3 shows that for an ideal mixture, $\bar{H}_{i}^{\mathrm{im}}(T, P, \mathbf{n})=h_{i}(T, P)$. We will later use this relation to calculate the enthalpy of real fluid mixtures. It is important to note that $\left(T, P, \mathbf{n}_{i}\right)$ must be held fixed in the derivative for it to be considered a partial molar property. For example, we will see in Section B.3 that $\mu_{i}=\left.\left(\partial F / \partial n_{i}\right)\right|_{T, V, \mathbf{n}_{i}}$, where $F$ is the Helmholtz energy of a fluid. Since it is the volume, and not the pressure, that is held fixed we conclude that $\mu_{i} \neq \bar{F}_{i}$. Instead, the partial molar Helmholtz energy is defined as

$$
\bar{F}_{i}=\left.\left(\frac{\partial F}{\partial n_{i}}\right)\right|_{T, P, \mathbf{n}_{i}} .
$$

We can also define partial molar properties of excess properties. For example,

$$
\bar{G}_{i}^{\text {excess }}=\left.\left(\frac{\partial G^{\text {excess }}}{\partial n_{i}}\right)\right|_{T, P, \mathbf{n}_{i}}=\mu_{i}(T, P, \mathbf{n})-\mu_{i}^{\mathrm{im}}(T, P, \mathbf{n}) .
$$

Using this relation along with $(12)$ and $(19)-20)$, we have

$$
\bar{G}_{i}^{\text {excess }}=\mu_{i}(T, P, \mathbf{n})-\mu_{i}^{\mathrm{im}}(T, P, \mathbf{n})=R T \ln \frac{f_{i}(T, P, \mathbf{n})}{f_{i}^{\mathrm{im}}(T, P, \mathbf{n})}=R T \ln \frac{z_{i} \gamma_{i} f_{i}(T, P)}{z_{i} f_{i}(T, P)}=R T \ln \gamma_{i} .
$$

This result shows that if we have a smooth function (such as a polynomial) that describes the variation of the excess Gibbs energy with the composition, we can take composition derivatives 
(i.e., evaluate the partial molar excess Gibbs energy $\bar{G}_{i}^{\text {excess }}$ ) to obtain the activity coefficient $\gamma_{i}$. In turn, we can use $\gamma_{i}$ to compute the real fluid mixture fugacity $f_{i}(T, P, \mathbf{n})$ from the pure component fugacity $f_{i}(T, P)$ using $(20)$. The smooth function is called the activity coefficient model. It is essentially a correlation which provides a continuous fit to a discrete set of excess Gibbs energy data, which are obtained through experimental measurements at different compositions. See [34, 37] for extensive discussions on activity coefficient models for various types of mixtures. Duan and Sun have presented a model for the $\mathrm{CO}_{2}$ activity coefficient in mixtures where $\mathrm{CO}_{2}$ is dissolved in aqueous sodium chloride solutions [3]. This model is partly based on the activity coefficient model of Pitzer, which is widely used to compute the excess Gibbs energy of electrolytic solutions [38, 34]. The Duan and Sun model is the focus of Section 3.2 and Appendix C.

\section{A.3 Enthalpy and density}

We stated in Section A.1 that the enthalpy of a fluid mixture can be derived from the fugacity coefficients $\phi_{i}$. To show this, we use the relation $G=H-T S$ between the Gibbs energy $G$ and the enthalpy $H$ so that the partial molar properties are related by

$$
\bar{G}_{i}=\mu_{i}=\bar{H}_{i}-T \bar{S}_{i} .
$$

From (8), we have

$$
\bar{S}_{i}=\left.\left(\frac{\partial S}{\partial n_{i}}\right)\right|_{T, P, \mathbf{n}_{i}}=-\left.\left(\frac{\partial \mu_{i}}{\partial T}\right)\right|_{P, \mathbf{n}}
$$

which leads to

$$
\mu_{i}=\bar{H}_{i}+\left.T\left(\frac{\partial \mu_{i}}{\partial T}\right)\right|_{P, \mathbf{n}}
$$

This result can be rearranged to

$$
\left.\frac{\partial}{\partial T}\left(\frac{\mu_{i}}{T}\right)\right|_{P, \mathbf{n}}=-\frac{\bar{H}_{i}}{T^{2}} .
$$

Since an ideal mixture is defined by (18),

$$
-\frac{\bar{H}_{i}^{\mathrm{im}}}{T^{2}}=\left.\frac{\partial}{\partial T}\left(\frac{\mu_{i}^{\mathrm{im}}}{T}\right)\right|_{P, \mathbf{n}}=\left.\frac{\partial}{\partial T}\left(\frac{\mu_{i}(T, P)+R T \ln z_{i}}{T}\right)\right|_{P, \mathbf{n}}=\left.\frac{\partial}{\partial T}\left(\frac{\mu_{i}(T, P)}{T}\right)\right|_{P, \mathbf{n}}=-\frac{h_{i}(T, P)}{T^{2}} .
$$

This is the result we alluded to in Section A.2 the partial molar enthalpy $\bar{H}_{i}^{\text {im }}$ is equal to the molar enthalpy $h_{i}(T, P)$ of the pure component. The enthalpy of ideal mixtures, which includes ideal gas mixtures, can thus be computed from the pure component enthalpies. In other words, ideal mixtures are characterized by zero enthalpies of mixing:

$$
\Delta h_{\mathrm{mix}}^{\mathrm{im}}=h^{\mathrm{im}}(T, P, \mathbf{z})-\sum_{i=1}^{c} z_{i} h_{i}(T, P)=\sum_{i=1}^{c} z_{i}\left(\bar{H}_{i}^{\mathrm{im}}-h_{i}\right)=0 .
$$

Applying (28) and (29) to (14), we have 


$$
\left.\frac{\partial}{\partial T}\left(\frac{\mu_{i}-\mu_{i}^{\mathrm{ig}}}{T}\right)\right|_{P, \mathbf{n}}=\left.R\left(\frac{\partial \ln \phi_{i}(T, P, \mathbf{n})}{\partial T}\right)\right|_{P, \mathbf{n}}=-\frac{\bar{H}_{i}-\bar{H}_{i}^{\mathrm{ig}}}{T^{2}}=-\frac{\bar{H}_{i}-h_{i}^{\mathrm{ig}}}{T^{2}} .
$$

Combining this result with $(26)$, the molar enthalpy of any fluid mixture is

$$
h(T, P, \mathbf{z})=\sum_{i=1}^{c} z_{i} \bar{H}_{i}=-\left.R T^{2} \sum_{i=1}^{c} z_{i}\left(\frac{\partial \ln \phi_{i}(T, P, \mathbf{n})}{\partial T}\right)\right|_{P, \mathbf{n}}+\sum_{i=1}^{c} z_{i} h_{i}^{\text {ig }} .
$$

This is the relation we seek between the fugacity coefficients and the enthalpy of the mixture. We can calculate the enthalpies $h_{i}^{\text {ig }}$ of the pure components in their ideal gas states using correlations found in the references specified in Section 2.

The density can be derived from the fugacity coefficients as well. Using (9) along with (14) and (26), the molar volume $v=V / N$ is computed from the partial molar volumes $\bar{V}_{i}$ according to

$$
v=\sum_{i=1}^{c} z_{i} \bar{V}_{i}=\left.\sum_{i=1}^{c} z_{i}\left(\frac{\partial \mu_{i}}{\partial P}\right)\right|_{T, \mathbf{n}}=\left.\sum_{i=1}^{c} z_{i}\left(\frac{\partial \mu_{i}^{\mathrm{ig}}}{\partial P}\right)\right|_{T, \mathbf{n}}+\left.R T \sum_{i=1}^{c} z_{i}\left(\frac{\partial \ln \phi_{i}}{\partial P}\right)\right|_{T, \mathbf{n}}
$$

For an ideal gas where $V^{\text {ig }}=N R T / P$

$$
\left.\left(\frac{\partial \mu_{i}^{\mathrm{ig}}}{\partial P}\right)\right|_{T, \mathbf{n}}=\bar{V}_{i}^{\mathrm{ig}}=\left.\left(\frac{\partial V^{\mathrm{ig}}}{\partial n_{i}}\right)\right|_{T, P, \mathbf{n}_{i}}=\frac{R T}{P} .
$$

Therefore,

$$
v=R T \sum_{i=1}^{c} z_{i}\left[\frac{1}{P}+\left.\left(\frac{\partial \ln \phi_{i}}{\partial P}\right)\right|_{T, \mathbf{n}}\right]
$$

and the molar density $\rho$ is

$$
\rho=\frac{1}{v}=\frac{1}{R T \sum_{i=1}^{c} z_{i}\left[\frac{1}{P}+\left.\left(\frac{\partial \ln \phi_{i}}{\partial P}\right)\right|_{T, \mathbf{n}}\right]}
$$

The mass density is

$$
\rho_{\text {mass }}=\rho \sum_{i=1}^{c} z_{i} M_{i},
$$

where $M_{i}$ is the molecular weight of component $i$.

\section{B Cubic-plus-association (CPA) equation of state}

\section{B.1 Intermolecular forces and departure functions}

It was stated at the end of Section A.1 that the fundamental role of an equation of state (EOS) is to provide an expression from which all other thermodynamic functions of a fluid can be derived. For equations of state that model complicated fluids like mixtures of $\mathrm{CO}_{2}$ and water, this expression is often in the form of a departure function [37, 33], which is sometimes also referred to as a residual 
or a residual function [34, 35]. A departure function is defined as the difference in some property (e.g., Helmholtz energy) of a real fluid and that same property of the fluid if it were to exist as an ideal gas mixture. This definition resembles the definition of an excess property in (24), but there are two important differences. First, the reference fluid in (24) is an ideal mixture, while it is an ideal gas mixture in a departure function. Second, excess properties are always defined as differences in some property $E$ for two different fluids at the same temperature, pressure, and composition $(T, P, \mathbf{n})$. In contrast, departure functions can involve two different fluids at the same $(T, P, \mathbf{n})$, or two fluids at the same $(T, V, \mathbf{n})$. The two types of departure functions are not necessarily the same. An ideal gas is by definition composed of non-interacting molecules represented by volumeless entities (points). In a real fluid, the departure from ideality arises because the molecules have a non-zero volume and interact with each other through intermolecular forces. The volume of the molecules is itself a reflection of repulsive intermolecular forces that have their origins in electrostatic forces and quantum mechanical effects (i.e., the Pauli exclusion principle) [39].

In order to understand the physical behavior represented by the CPA EOS, and indeed equations of state in general, we briefly review some basic concepts regarding intermolecular forces. The discussion here will also motivate the future work described in Sections 4 and 5 . Intermolecular interactions can generally be divided into two sets: physical and chemical/quasi-chemical. Physical interactions include London dispersion forces (which can be thought of as instantaneous dipoleinduced dipole interactions), dipole-dipole interactions (also called Keesom forces), and dipoleinduced dipole interactions (Debye forces). They are sometimes collectively referred to as van der Waals forces [26]. On a per-mole basis, the van der Waals forces are weaker than other types of interactions. However, because they form rather easily, especially London dispersion forces, they are relatively numerous in a given fluid and can significantly influence the properties of a fluid.

Chemical interactions include hydrogen bonds, which act between a molecule that contains the electronegative atoms oxygen $(\mathrm{O})$, nitrogen $(\mathrm{N})$, or fluorine $(\mathrm{F})$ bound to hydrogen $(\mathrm{H})$ and another molecule that contains $\mathrm{O}, \mathrm{N}$, or $\mathrm{F}$. The hydrogen develops a partial positive charge, while the electronegative atom to which it is covalently bonded develops partial negative charge. Hydrogen bonding allows molecules of a fluid to associate to form polymers. For example, methanol consists of $\mathrm{CH}_{3} \mathrm{OH}$ monomers, some of which polymerize to form $\mathrm{CH}_{3} \mathrm{OH}$ dimers, trimers, or longer-chained polymers. The monomer units are linked together (associated) by hydrogen bonds between the oxygen on a methanol molecule and the hydrogen in the hydroxyl group of another methanol. Hydrogen bonds are classified as chemical interactions because they are quite strong and resemble chemical (i.e., covalent) bonds in that they involve partial overlap of the electron clouds of the atoms involved in the interaction [34]. In contrast, physical interactions do not involve significant overlap of electron clouds.

Hydrogen bonding plays an essential role in determining water's unusual properties. Ice consists of a regular lattice of water molecules, with each molecule being bound to four other molecules through hydrogen bonds. Each molecule is said to have four associating sites (Figure 9), which come in two varieties: 2 hydrogen-bond donor $(\alpha)$ sites representing the two hydrogens, and 2 hydrogen-bond acceptor $(\beta)$ sites representing the two lone pairs of electrons on the oxygen. In liquid water, the molecules also form hydrogen bonds with each other, but these interactions are more transient; hydrogen bonds are constantly forming and breaking, and the molecules are able to move past each other. The regular, hydrogen-bonded lattice structure of ice limits the maximum packing achievable, which is why ice is less dense than liquid water at the normal melting point.

In fluid mixtures, the components can be either self-associating or non-self-associating, based on 


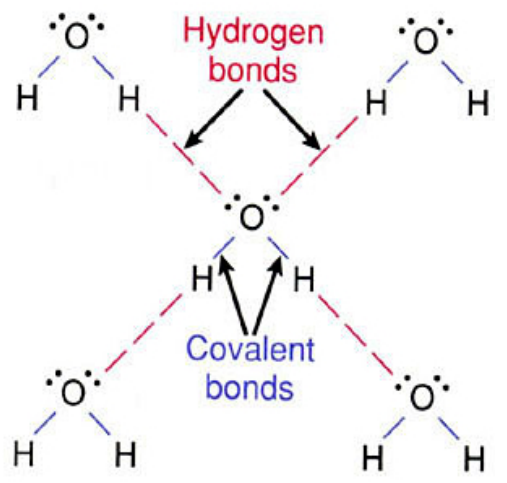

Figure 9: Hydrogen bonding in water. Each molecule can form up to four hydrogen bonds since it has four associating sites: 2 hydrogen-bond donor (also called electron acceptor) sites representing the two hydrogens, and 2 hydrogen-bond acceptor (electron donor) sites representing the two electron lone pairs on the oxygen. We will refer to these two site types as $\alpha$ and $\beta$ sites, respectively.

whether the pure component forms hydrogen bonds. In mixtures with at least one self-associating component, there may be cross-association between the self-associating component and the other components. For example, $\mathrm{CO}_{2}$ may cross-associate with water because the lone pairs on the oxygens of $\mathrm{CO}_{2}$ can serve as hydrogen-bond acceptor sites. Molecules without associating sites, such as $\mathrm{H}_{2} \mathrm{~S}$ or alkanes, may also cross-associate with water. In this case, the cross-association provides a simple way to implicitly account for the complicated physical and chemical processes that occur during the solvation of these molecules in water.

\section{B.2 Helmholtz energy departure function}

\section{B.2.1 Overview of Peng-Robinson and CPA EOS}

The Peng-Robinson (PR) EOS belongs to the family of cubic equations of state, which are all based on the van der Waals equation of state [33. The PR EOS is widely used in the oil and gas industry to model fluid mixtures containing only non-self-associating components, such as hydrocarbons. It, along with other cubic EOS variants like the Soave-Redlich-Kwong (SRK) EOS, involves an interaction parameter $a$, which accounts for van der Waals interactions, and a co-volume parameter $b$, which accounts for the non-zero volume of the fluid molecules. If $a=b=0$, the PR EOS reduces to the ideal gas EOS. Since $a$ accounts for only van der Waals interactions, the PR EOS in its original form cannot model fluid mixtures with associating components, such as water.

One approach to model mixtures with associating components is to append the PR EOS, or a different cubic EOS, with an additional term that accounts for the association. This approach forms the basis of the cubic-plus-association (CPA) EOS. The CPA EOS in its modern form was introduced by Kontogeorgis et al. [40], although earlier authors have developed a similar EOS [41]. Other models for associating fluids besides CPA are described in various studies [42, 43], and will not be considered further in this report. The cubic part of the CPA EOS by Kontogeorgis et al. comes from the SRK EOS. The association (hydrogen bonding) part is based on the thermodynamic perturbation theory of Wertheim [44, 45, 46, 47]. In fluids without associating components, the CPA EOS of Kontogeorgis et al. reduces to the SRK EOS. 
Li and Firoozabadi have presented a version of the CPA EOS where they introduce a novel scheme to model the cross-association between molecules of different components 2 . Their study concludes that cross-association is necessary to faithfully reproduce experimental vapor-liquid equilibria (VLE) data, especially along the vapor phase, for mixtures containing water and one or more of the following components: $\mathrm{CO}_{2}, \mathrm{H}_{2} \mathrm{~S}$, and hydrocarbons (both aliphatic chains and aromatics). This conclusion is in agreement with earlier studies, who also report that cross-association is important in water/alkane mixtures and water $/ \mathrm{CO}_{2}$ mixtures [48, 12. Li and Firoozabadi's original version of the CPA is targeted toward mixtures where water is the only self-associating component [2]. They later modified their EOS to model mixtures where asphaltenes serve as the self-associating component [49, 50]. The Li and Firoozabadi CPA EOS with water as the only self-associating component will be the focus of the present study.

The starting point is to consider the CPA EOS's expression for the departure function $F^{\text {depart }}$ of the Helmholtz energy. Since the Helmholtz energy $F$ is a natural function of $(T, V, \mathbf{n}), F^{\text {depart }}$ is defined as the difference between the Helmholtz energy of the real fluid and the Helmholtz energy of the corresponding ideal gas at the same $(T, V, \mathbf{n})$ :

$$
F^{\text {depart }}(T, V, \mathbf{n})=F(T, V, \mathbf{n})-F^{\mathrm{ig}}(T, V, \mathbf{n}) .
$$

Ideal gases by definition consist of non-interacting molecules of zero volume. The departure function is therefore a measure of the contribution to $F(T, V, \mathbf{n})$ from intermolecular forces plus the non-zero volume taken up by the molecules. These contributions can be divided into two categories

$$
F^{\text {depart }}=F_{\text {phys }}^{\text {depart }}+F_{\text {assoc }}^{\text {depart }}
$$

where $F_{\text {phys }}^{\text {depart }}$ is the contribution from physical (van der Waals) interactions and non-zero molecular volumes, and $F_{\text {assoc }}^{\text {depart }}$ is the contribution from association (hydrogen bonding). Note that because intermolecular forces like ion-dipole interactions and ion-ion interactions are not accounted for in the departure function, the CPA EOS cannot model mixtures involving brines, such as $\mathrm{H}_{2} \mathrm{O}_{-} \mathrm{CO}_{2^{-}}$ $\mathrm{NaCl}$ mixtures. It can model mixtures with $\mathrm{CO}_{2}$ and $\mathrm{H}_{2} \mathrm{O}$ only.

\section{B.2.2 Physical contribution to the Helmholtz energy departure function}

The physical contribution $F_{\text {phys }}^{\text {depart }}$ is modeled by the cubic part of the EOS. Unlike the original CPA introduced by Kontogeorgis et al., Li and Firoozabadi use the PR EOS, not the SRK EOS, for their cubic part. The expression for $F_{\text {phys }}^{\text {depart }}$ given by the PR EOS is

$$
\frac{F_{\text {phys }}^{\text {depart }}}{N R T}=-\ln (1-b \rho)-\frac{a}{2 \sqrt{2} b R T} \ln \left[\frac{1+(1+\sqrt{2}) b \rho}{1+(1-\sqrt{2}) b \rho}\right],
$$

where $a=a(T, \mathbf{z})$ is the temperature-dependent parameter that accounts for the physical interactions, and $b=b(\mathbf{z})$ is the temperature-independent parameter that accounts for non-zero molecular volumes. In mixtures, $a$ and $b$ are computed from the pure component properties $a_{i}$ and $b_{i}$ using the standard van der Waals mixing rules:

$$
\begin{aligned}
& a_{i j}=\left(1-k_{i j}\right) a_{i}^{1 / 2} a_{j}^{1 / 2}, \\
& a(T, \mathbf{z})=\sum_{i=1}^{c} \sum_{j=1}^{c} z_{i} z_{j} a_{i j},
\end{aligned}
$$




$$
b(\mathbf{z})=\sum_{i=1}^{c} z_{i} b_{i}
$$

where $k_{i j}=k_{i j}(T)$ is the binary interaction coefficient between component $i$ and $j$. This coefficient is fitted to experimental VLE data. Fits to binary water $/ \mathrm{CO}_{2}$ mixture data give

$$
k_{i j}=0.5994\left(T / T_{c, \mathrm{CO}_{2}}\right)-0.5088,
$$

for $k_{i j}$ between water and $\mathrm{CO}_{2}$ in which $T_{c, \mathrm{CO}_{2}}$ is the $\mathrm{CO}_{2}$ critical temperature. Correlations for $k_{i j}$ of non-self-associating components may be found in [51]. The pure component properties $a_{i}$ and $b_{i}$ in (34) and (35) are computed from three parameters if it is a non-self-associating: the reduced temperature $T_{r, i}$; the reduced pressure $P_{r, i}$; and the acentric factor $\omega_{i}$. They are defined as

$$
\begin{gathered}
T_{r, i}=T / T_{c, i}, \\
P_{r, i}=P / P_{c, i}, \\
\omega_{i}=-\left.\log _{10}\left(\frac{P_{i}^{\mathrm{vap}}}{P_{c, i}}\right)\right|_{T_{r, i}=0.7}-1,
\end{gathered}
$$

where $T_{c, i}$ is the critical temperature of component $i, P_{c, i}$ is its critical pressure, and $P_{i}^{\text {vap }}$ is its vapor pressure. For the acentric factor, the vapor pressure is evaluated at a temperature corresponding to $T_{r, i}=0.7$. For a non-self-associating component $i$ [36], its properties $a_{i}$ and $b_{i}$ are

$$
\begin{gathered}
a_{i}(T)=a_{c}\left(T_{c, i}\right) \alpha\left(\omega_{i}, T_{r, i}\right), \\
a_{c}\left(T_{c, i}\right)=0.45724 \frac{R^{2} T_{c, i}^{2}}{P_{c, i}}, \\
\alpha\left(\omega_{i}, T_{r, i}\right)=\left[1+m_{i}\left(1-\sqrt{T_{r, i}}\right)\right]^{2}, \\
m_{i}=\left\{\begin{array}{l}
0.3796+1.485 \omega_{i}-0.1644 \omega_{i}^{2}+0.01667 \omega_{i}^{3}, \quad 0.1<\omega_{i}<2.0, \\
0.37464+1.54226 \omega_{i}-0.26992 \omega_{i}^{2}, \quad \omega_{i}<0.1, \\
b_{i}=0.0778 \frac{R T_{c, i}}{P_{c, i}} .
\end{array}\right.
\end{gathered}
$$

For water (the self-associating component), $a_{\mathrm{H}_{2} \mathrm{O}}$ is computed from the correlation suggested by Mathias et al. [52]:

$$
a_{\mathrm{H}_{2} \mathrm{O}}(T)=a_{0}\left[1+c_{1}\left(1-\sqrt{T_{r, \mathrm{H}_{2} \mathrm{O}}}\right)+c_{2}\left(1-\sqrt{T_{r, \mathrm{H}_{2} \mathrm{O}}}\right)^{2}+c_{3}\left(1-\sqrt{T_{r, \mathrm{H}_{2} \mathrm{O}}}\right)^{3}\right],
$$

where $a_{0}, c_{1}, c_{2}, c_{3}$ are constants. Li and Firoozabadi have found that choosing $c_{1}=1.7557, c_{2}=$ $0.003518, c_{3}=-0.2746$, and $a_{0}=0.9627 \mathrm{~L}^{2} \cdot \mathrm{bar} / \mathrm{mole}^{2}$ provides a good match with experimental data. The volume parameter of water is $b_{\mathrm{H}_{2} \mathrm{O}}=0.01458 \mathrm{~L} /$ mole. Equation (33) is commonly expressed in terms of the compressibility factor $Z$

$$
Z=\frac{P V}{N R T}=\frac{P}{\rho R T},
$$


by defining new variables $A$ and $B$ that are analogues to $a$ and $b$, respectively:

$$
\begin{gathered}
A(T, P, \mathbf{z})=\frac{a P}{R^{2} T^{2}}, \\
B(T, P, \mathbf{z})=\frac{b P}{R T} .
\end{gathered}
$$

Substituting (36)-(38) into (33) yields

$$
\frac{F_{\mathrm{phys}}^{\text {depart }}}{N R T}=\ln \left(\frac{Z}{Z-B}\right)-\frac{A}{2 \sqrt{2} B} \ln \left[\frac{Z+(1+\sqrt{2}) B}{Z+(1-\sqrt{2}) B}\right] .
$$

\section{B.2.3 Association contribution to the Helmholtz energy departure function}

As mentioned in Section B.1, there are two types of association sites: hydrogen-bond donor sites, which we will denote as $\alpha$ sites, and hydrogen-bond acceptor sites, which we will denote as $\beta$ sites. Hydrogen bonds form only between sites of opposite types; $\alpha(\beta)$ sites on a molecule can associate with only the $\beta(\alpha)$ sites on another molecule. The general expression for $F_{\text {assoc }}^{\text {depart }}$ is

$$
\frac{F_{\text {assoc }}^{\text {depart }}}{N R T}=\sum_{i=1}^{c} z_{i} \sum_{j=\alpha, \beta} \eta_{i j}\left(\ln \chi_{i j}-\frac{\chi_{i j}-1}{2}\right),
$$

where $\eta_{i j}$ is the number of $j$ sites $\left(j=\alpha\right.$ or $\beta$ ) on a molecule of component $i$, and $\chi_{i j}$ is the fraction of component $i$ molecules whose $j$ sites are not associated (i.e., $j$ sites that are still free to form hydrogen bonds) [43. We make the following key assumption: water is the only self-associating component in the mixture. Other components may cross-associate with water, but they do not self-associate nor do they cross-associate with each other. This means that in addition to being inapplicable to electrolytic solutions [see discussion below Equation (32)], the CPA EOS that we consider is also limited to mixtures where water is the only self-associating component. It would not be applicable to aqueous mixtures of alcohols or amines, for example. Nonetheless, this assumption is sufficient for $\mathrm{CO}_{2}-\mathrm{H}_{2} \mathrm{O}$ mixtures, since $\mathrm{CO}_{2}$ lacks the hydrogen atoms that are necessary for self-association. The site fractions for water (denoted by the subscript ' $w$ '), are obtained by solving equations suggested by Kontogeorgis et al. [40]:

$$
\begin{aligned}
\chi_{w \alpha} & =\frac{1}{1+\rho \sum_{i=1}^{c} z_{i} \eta_{i \beta} \chi_{i \beta} \delta_{w \alpha, i \beta}}, \\
\chi_{w \beta} & =\frac{1}{1+\rho \sum_{i=1}^{c} z_{i} \eta_{i \alpha} \chi_{i \alpha} \delta_{w \beta, i \alpha}},
\end{aligned}
$$

in which $\delta_{w \alpha, i \beta}$ is the association strength between an $\alpha$ site on a water molecule and a $\beta$ site on a molecule of component $i$. The quantity $\delta_{w \beta, i \alpha}$ is defined similarly. Equations (41) and (42) essentially state that the fraction of non-associated $\alpha(\beta)$ sites on water, as expressed by $\chi_{w \alpha}$ $\left(\chi_{w \beta}\right)$, decreases with the density of available, meaning non-associated, $\beta(\alpha)$ sites on each component $i$, which is given by $\rho z_{i} \eta_{i \beta} \chi_{i \beta}\left(\rho z_{i} \eta_{i \alpha} \chi_{i \alpha}\right)$, and with the association strength of $\beta(\alpha)$ sites on component $i$. The association strengths are defined according to

$$
\delta_{w \alpha, w \beta}=\delta_{w \beta, w \alpha}=g \kappa\left[\exp \left(\frac{\epsilon}{k_{B} T}\right)-1\right]
$$




$$
\begin{aligned}
& \delta_{w \alpha, i \beta}=s_{w \alpha, i \beta} \delta_{w \alpha, w \beta} \quad i \neq w, \\
& \delta_{w \beta, i \alpha}=s_{w \beta, i \alpha} \delta_{w \beta, w \alpha} \quad i \neq w,
\end{aligned}
$$

where $s_{w \alpha, i \beta}=s_{w \alpha, i \beta}(T)$ is the parameter of cross-association between an $\alpha$ site on water with a $\beta$ site on component $i$, and $s_{w \beta, i \alpha}$ is defined similarly. The cross-association parameters are expressed as second-order polynomials of the reduced temperature $T_{r, i}$ whose coefficients can be fitted to experimental data [2]. The energy and volume parameters of water self-association are denoted by $\epsilon$ and $\kappa$, respectively. Their values are

$$
\begin{gathered}
\frac{\epsilon}{k_{B}}=1738.4 \mathrm{~K}, \\
\kappa=1.8015 \times 10^{-3} \frac{\mathrm{L}}{\text { mole }},
\end{gathered}
$$

where $k_{B}$ is the Boltzmann constant. These parameters have been fitted to data for pure water. Finally, $g=g(\eta)$ in (43) represents the contact value of the radial distribution function for water. We may denote this function with its argument $\eta$ to distinguish it from the molar Gibbs energy $g(T, P, \mathbf{z})$. Li and Firoozabadi [2] use the expression for $g(\eta)$ from the Starling-Carnahan EOS, which states

$$
\begin{gathered}
g(\eta)=\frac{1-0.5 \eta}{(1-\eta)^{3}} \\
\eta=\frac{b \rho}{4}=\frac{B}{4 Z} .
\end{gathered}
$$

Studies have found expressions for $g(\eta)$ simpler than that in 47) also work well [48, but we continue to use 47 to be consistent with the work of Li and Firoozabadi. Since water is the only self-associating component and other components may cross-associate only with water, as stated at the beginning of this section, the site fractions for these components are

$$
\begin{aligned}
\chi_{i \alpha} & =\frac{1}{1+\rho z_{w} \eta_{w \beta} \chi_{w \beta} \delta_{w \beta, i \alpha}}, \\
\chi_{i \beta} & =\frac{1}{1+\rho z_{w} \eta_{w \alpha} \chi_{w \alpha} \delta_{w \alpha, i \beta}} .
\end{aligned}
$$

Note that in non-associating fluids, the association strengths are all zero so that from (41)- 42) and (49)-(50), all site fractions become zero and the CPA EOS reduces to the PR EOS. In summary, the association contribution to the Helmholtz energy departure function is given by 40 , where the site fractions are obtained by solving $41-(42)$ and $(49)-(50)$. The association strengths that affect the site fractions are calculated using (43)-(48). Substituting (33) and (40) into (32), we obtain the full expression for the Helmholtz energy departure function

$$
\begin{aligned}
\frac{F^{\text {depart }}}{N R T}=-\ln (1-b \rho) & -\frac{a}{2 \sqrt{2} b R T} \ln \left[\frac{1+(1+\sqrt{2}) b \rho}{1+(1-\sqrt{2}) b \rho}\right] \\
& +\sum_{i=1}^{c} z_{i} \sum_{j=\alpha, \beta} \eta_{i j}\left(\ln \chi_{i j}-\frac{\chi_{i j}-1}{2}\right) .
\end{aligned}
$$


Section B.4 will derive the expression for the pressure from (51). This form of $F^{\text {depart }}$ is useful for this purpose because $P$ does not appear explicitly. However, for the purposes of the fugacity coefficient $\phi_{i}$, which we derive in the Section B.3. it is more convenient to express $F^{\text {depart }}$ in terms of the compressibility factor $Z=P / \rho R T$. To do so, we define new expressions for the association strengths according to

$$
\begin{gathered}
\mathcal{K}=\frac{\kappa P}{R T}, \\
\Delta_{w \alpha, w \beta}=\Delta_{w \beta, w \alpha}=g \mathcal{K}\left[\exp \left(\frac{\epsilon}{k_{B} T}\right)-1\right], \\
\Delta_{w \alpha, i \beta}=s_{w \alpha, i \beta} \Delta_{w \alpha, w \beta} \quad i \neq w \\
\Delta_{w \beta, i \alpha}=s_{w \beta, i \alpha} \Delta_{w \beta, w \alpha} \quad i \neq w .
\end{gathered}
$$

Note that $\Delta_{i j, k l}=(P / R T) \delta_{i j, k l}$ for all $i, j, k, l$. The site fractions are given by

$$
\begin{gathered}
\chi_{w \alpha}=\frac{Z}{Z+\sum_{i=1}^{c} z_{i} \eta_{i \beta} \chi_{i \beta} \Delta_{w \alpha, i \beta}}, \\
\chi_{w \beta}=\frac{Z}{Z+\sum_{i=1}^{c} z_{i} \eta_{i \alpha} \chi_{i \alpha} \Delta_{w \beta, i \alpha}}, \\
\chi_{i \alpha}=\frac{Z}{Z+z_{w} \eta_{w \beta} \chi_{w \beta} \Delta_{w \beta, i \alpha}}, \\
\chi_{i \beta}=\frac{Z}{Z+z_{w} \eta_{w \alpha} \chi_{w \alpha} \Delta_{w \alpha, i \beta}} .
\end{gathered}
$$

In terms of $Z$, the association contribution to the Helmholtz energy departure function is given by (40), where the site fractions are obtained by solving (56)-(59). The association strengths that

affect the site fractions are calculated using (52)-(55) and (46)-(48). Substituting (39) and (40) into (32), we obtain the full expression for the Helmholtz energy departure function

$$
\frac{F^{\text {depart }}}{N R T}=\ln \left(\frac{Z}{Z-B}\right)-\frac{A}{2 \sqrt{2} B} \ln \left[\frac{Z+(1+\sqrt{2}) B}{Z+(1-\sqrt{2}) B}\right]+\sum_{i=1}^{c} z_{i} \sum_{j=\alpha, \beta} \eta_{i j}\left(\ln \chi_{i j}-\frac{\chi_{i j}-1}{2}\right)
$$

\section{B.3 Fugacity coefficient, enthalpy, density}

In this section, we derive the expression for the fugacity coefficient $\phi_{i}$ from the Helmholtz energy departure function (60). The fugacity coefficient is an important quantity in phase equilibria (solubility) calculations, as discussed in Section A.1. The fugacity coefficient can also be used to compute the enthalpy and the density, as described in Section A.3. We start with the expression for the differential of the Helmholtz energy $F$. For systems where external fields like gravity are negligible and there is only pressure-volume work,

$$
\mathrm{d} F=-S \mathrm{~d} T-P \mathrm{~d} V+\sum_{i=1}^{c} \mu_{i} \mathrm{~d} n_{i}
$$


from which it follows that

$$
\mu_{i}=\left.\left(\frac{\partial F}{\partial n_{i}}\right)\right|_{T, V, \mathbf{n}_{i}} .
$$

This expression, when combined with the definition of $F^{\text {depart }}$ in $(31)$, leads to

$$
\frac{\mu_{i}(T, V, \mathbf{n})-\mu_{i}^{\mathrm{ig}}(T, V, \mathbf{n})}{R T}=\left.\frac{1}{R T}\left(\frac{\partial F^{\text {depart }}}{\partial n_{i}}\right)\right|_{T, V, \mathbf{n}_{i}} .
$$

The left-hand side of (62) is similar to the left-hand side of (14), except that the ideal gas in (14) is at the same pressure (but not same volume) as the real fluid, while the ideal gas in (62) is at the same volume (but not same pressure) as the real fluid. We can denote the volume of the ideal gas in (14) as $V^{\mathrm{ig}}=N R T / P$ so that its chemical potential is $\mu_{i}^{\mathrm{ig}}(T, P, \mathbf{n})=\mu_{i}^{\mathrm{ig}}\left(T, V^{\mathrm{ig}}, \mathbf{n}\right)$. Similarly, the pressure of the ideal gas in $(62)$ is $P^{\mathrm{ig}}=N R T / V$ so that its chemical potential is $\mu_{i}^{\mathrm{ig}}(T, V, \mathbf{n})=\mu_{i}^{\mathrm{ig}}\left(T, P^{\mathrm{ig}}, \mathbf{n}\right)$. The two different ideal gas states are related by

$$
\mu_{i}^{\mathrm{ig}}(T, V, \mathbf{n})-\mu_{i}^{\mathrm{ig}}\left(T, V^{\mathrm{ig}}, \mathbf{n}\right)=\left.\int_{V^{\mathrm{ig}}}^{V}\left(\frac{\partial \mu_{i}^{\mathrm{ig}}}{\partial V^{\prime}}\right)\right|_{T, \mathbf{n}} \mathrm{d} V^{\prime}
$$

From the equality of mixed derivatives in (61), we readily obtain

$$
-\left.\left(\frac{\partial P}{\partial n_{i}}\right)\right|_{T, V, \mathbf{n}_{i}}=\left.\left(\frac{\partial \mu_{i}}{\partial V}\right)\right|_{T, \mathbf{n}}
$$

which we apply to the integral

$$
\left.\int_{V^{\mathrm{ig}}}^{V}\left(\frac{\partial \mu_{i}^{\mathrm{ig}}}{\partial V^{\prime}}\right)\right|_{T, \mathbf{n}} \mathrm{d} V^{\prime}=-\left.\int_{V^{\mathrm{ig}}}^{V}\left(\frac{\partial P^{\mathrm{ig}}}{\partial n_{i}}\right)\right|_{T, V^{\prime}, \mathbf{n}_{i}} \mathrm{~d} V^{\prime}=R T \ln \left(\frac{V^{\mathrm{ig}}}{V}\right)=-R T \ln Z .
$$

Thus,

$$
\mu_{i}(T, V, \mathbf{n})-\mu_{i}^{\mathrm{ig}}(T, V, \mathbf{n})=\mu_{i}(T, P, \mathbf{n})-\left[\mu_{i}^{\mathrm{ig}}(T, P, \mathbf{n})-R T \ln Z\right],
$$

so that combining with (14) and (62), we obtain

$$
\ln \phi_{i}=\left.\frac{1}{R T}\left(\frac{\partial F^{\text {depart }}}{\partial n_{i}}\right)\right|_{T, V, \mathbf{n}_{i}}-\ln Z .
$$

Since the departure function can be divided into a physical contribution and an association contribution [see Equation (32)], we can also divide the fugacity coefficient into a physical contribution and an association contribution according to

$$
\ln \phi_{i}^{\text {phys }}=\left.\frac{1}{R T}\left(\frac{\partial F_{\text {phys }}^{\text {depart }}}{\partial n_{i}}\right)\right|_{T, V, \mathbf{n}_{i}}-\ln Z,
$$

and

$$
\ln \phi_{i}^{\mathrm{assoc}}=\left.\frac{1}{R T}\left(\frac{\partial F_{\mathrm{assoc}}^{\mathrm{depart}}}{\partial n_{i}}\right)\right|_{T, V, \mathbf{n}_{i}}
$$


It can be shown (see for example, [37]) that the fugacity coefficient of the PR EOS is

$$
\begin{aligned}
\ln \phi_{i}^{\text {phys }}=-\ln (Z-B) & +\frac{B_{i}}{B}\left(\frac{B}{Z-B}-\frac{A Z}{Z^{2}+2 B Z-B^{2}}\right) \\
& -\frac{A}{2 \sqrt{2} B}\left(\frac{2 \sum_{j=1}^{c} z_{j} A_{i j}}{A}-\frac{B_{i}}{B}\right) \ln \left[\frac{Z+(1+\sqrt{2}) B}{Z+(1-\sqrt{2}) B}\right],
\end{aligned}
$$

where

$$
\begin{gathered}
B_{i}=\frac{b_{i} P}{R T}, \\
A_{i j}=\frac{a_{i j} P}{R^{2} T^{2}}=\frac{\left(1-k_{i j}\right) a_{i}^{1 / 2} a_{j}^{1 / 2} P}{R^{2} T^{2}} .
\end{gathered}
$$

Equation (64) is difficult to evaluate because the site fractions $\chi=\left(\chi_{1 \alpha}, \chi_{1 \beta}, \chi_{2 \alpha}, \ldots, \chi_{c \beta}\right)$ are implicit functions of each other [see (56)-59]], and thus, it is not immediately clear how to evaluate their derivatives. We avoid having to evaluate the site-fraction derivatives by employing the method developed by Michelsen and Hendriks [53]. Their method involves constructing a function $Q=Q(T, V, \mathbf{n}, \boldsymbol{\chi})$ such that $Q$ is equal to $F_{\text {assoc }}^{\text {depart }} / R T$ at a stationary point (labeled 'sp') with respect to the site fractions $\chi$. That is, $Q=Q_{\mathrm{sp}}=F_{\mathrm{assoc}}^{\text {depart }} / R T$ at a point where

$$
\left.\left(\frac{\partial Q_{\mathrm{sp}}}{\partial \chi_{i j}}\right)\right|_{\left(T, V, \mathbf{n}, \chi_{i j}\right)}=0 \text { for all } \chi_{i j}
$$

The subscript $\chi_{i j}$ in the derivative above means that all site fractions besides $\chi_{i j}$ are held fixed. Therefore,

$$
\ln \phi_{i}^{\text {assoc }}=\left.\frac{1}{R T}\left(\frac{\partial F_{\mathrm{assoc}}^{\text {depart }}}{\partial n_{i}}\right)\right|_{T, V, \mathbf{n}_{i}}=\left.\left(\frac{\partial Q_{\mathrm{sp}}}{\partial n_{i}}\right)\right|_{T, V, \mathbf{n}_{i}} .
$$

Applying the chain rule,

$$
\left.\left(\frac{\partial Q_{\mathrm{sp}}}{\partial n_{i}}\right)\right|_{T, V, \mathbf{n}_{i}}=\left.\left(\frac{\partial Q_{\mathrm{sp}}}{\partial n_{i}}\right)\right|_{T, V, \mathbf{n}_{i}, \chi}+\left.\left.\sum_{i=1}^{c} \sum_{j=\alpha, \beta}\left(\frac{\partial Q_{\mathrm{sp}}}{\partial \chi_{i j}}\right)\right|_{\left(T, V, \mathbf{n}, \chi_{i j}\right)}\left(\frac{\partial \chi_{i j}}{\partial n_{i}}\right)\right|_{T, V, \mathbf{n}_{i}} .
$$

By definition of the stationary point in (68), Equation (69) simplifies to

$$
\left.\left(\frac{\partial Q_{\mathrm{sp}}}{\partial n_{i}}\right)\right|_{T, V, \mathbf{n}_{i}}=\left.\left(\frac{\partial Q_{\mathrm{sp}}}{\partial n_{i}}\right)\right|_{T, V, \mathbf{n}_{i}, \chi}
$$

The site fractions $\chi$ are held constant in 70 . The result of all this is that the troublesome derivatives $\left.\left(\partial \chi_{i j} / \partial n_{i}\right)\right|_{T, V, \mathbf{n}_{i}}$ do not need to be evaluated. The task remains to select a good choice for the function $Q$. One such choice is 


$$
\begin{aligned}
Q(T, V, \mathbf{n}, \boldsymbol{\chi})= & \sum_{i=1}^{c} n_{i} \sum_{j=\alpha, \beta} \eta_{i j}\left(\ln \chi_{i j}-\chi_{i j}+1\right) \\
& -\frac{1}{2 V} \sum_{i=1}^{c} n_{i} \sum_{j=\alpha, \beta} \eta_{i j} \chi_{i j} \sum_{k=1}^{c} n_{k} \sum_{l=\alpha, \beta} \eta_{k l} \chi_{k l} \delta_{i j, k l} .
\end{aligned}
$$

It must first be shown that $Q_{\mathrm{sp}}=F_{\mathrm{assoc}}^{\text {depart }} / R T$. At a stationary point,

$$
\left.\left(\frac{\partial Q_{\mathrm{sp}}}{\partial \chi_{i j}}\right)\right|_{\left(T, V, \mathbf{n}, \chi_{i j}\right)}=n_{i} \eta_{i j}\left(\frac{1}{\chi_{i j}}-1\right)-\frac{n_{i} \eta_{i j}}{V} \sum_{k=1}^{c} n_{k} \sum_{l=\alpha, \beta} \eta_{k l} \chi_{k l} \delta_{i j, k l}=0 .
$$

Rearranging, we have

$$
\frac{1}{V} \sum_{k=1}^{c} n_{k} \sum_{l=\alpha, \beta} \eta_{k l} \chi_{k l} \delta_{i j, k l}=\frac{1}{\chi_{i j}}-1
$$

Substituting this result into (71) and comparing with (40), we see (since $\left.z_{i}=n_{i} / N\right)$ that

$$
Q_{\mathrm{sp}}=\sum_{i=1}^{c} n_{i} \sum_{j=\alpha, \beta} \eta_{i j}\left(\ln \chi_{i j}-\frac{\chi_{i j}-1}{2}\right)=\frac{F_{\text {assoc }}^{\text {depart }}}{R T} .
$$

Substituting (71) into (70), we have

$$
\begin{aligned}
\left.\left(\frac{\partial Q_{\mathrm{sp}}}{\partial n_{m}}\right)\right|_{T, V, \mathbf{n}_{m}, \chi}= & \sum_{j=\alpha, \beta} \eta_{m j}\left(\ln \chi_{m j}-\chi_{m j}+1\right)-\frac{\sum_{j=\alpha, \beta} \eta_{m j} \chi_{m j}}{V} \sum_{k=1}^{c} n_{k} \sum_{l=\alpha, \beta} \eta_{k l} \chi_{k l} \delta_{m j, k l} \\
& -\left.\frac{1}{2 V} \sum_{i=1}^{c} n_{i} \sum_{j=\alpha, \beta} \eta_{i j} \chi_{i j} \sum_{k=1}^{c} n_{k} \sum_{l=\alpha, \beta} \eta_{k l} \chi_{k l}\left(\frac{\partial \delta_{i j, k l}}{\partial n_{m}}\right)\right|_{T, V, \mathbf{n}_{m}, \chi} .
\end{aligned}
$$

Using (72), this simplifies to

$$
\begin{aligned}
\left.\left(\frac{\partial Q_{\mathrm{sp}}}{\partial n_{m}}\right)\right|_{T, V, \mathbf{n}_{m}, \chi}= & \sum_{j=\alpha, \beta} \eta_{m j} \ln \chi_{m j} \\
& -\left.\frac{1}{2 V} \sum_{i=1}^{c} n_{i} \sum_{j=\alpha, \beta} \eta_{i j} \chi_{i j} \sum_{k=1}^{c} n_{k} \sum_{l=\alpha, \beta} \eta_{k l} \chi_{k l}\left(\frac{\partial \delta_{i j, k l}}{\partial n_{m}}\right)\right|_{T, V, \mathbf{n}_{m}, \chi} .
\end{aligned}
$$

From (43), we see that the only part of $\delta_{i j, k l}$ that depends on the mole numbers is the contact value of the radial distribution function $g(\eta)$. Therefore, using (48) yields

$$
\left.\left(\frac{\partial \delta_{i j, k l}}{\partial n_{m}}\right)\right|_{T, V, \mathbf{n}_{m}, \chi}=\left.\frac{\delta_{i j, k l}}{g} \frac{\mathrm{d} g}{\mathrm{~d} \eta}\left(\frac{\partial \eta}{\partial n_{m}}\right)\right|_{T, V, \mathbf{n}_{m}, \chi}=\frac{\delta_{i j, k l}}{g} \frac{\mathrm{d} g}{\mathrm{~d} \eta} \frac{b_{m}}{4 V}=\frac{\delta_{i j, k l}}{g} \frac{\mathrm{d} g}{\mathrm{~d} \eta} \frac{B_{m}}{4 Z} \frac{1}{N},
$$

where

$$
\frac{\mathrm{d} g}{\mathrm{~d} \eta}=\frac{2.5-\eta}{(1-\eta)^{4}}
$$


Substituting into the above, we have

$$
\begin{aligned}
\left.\left(\frac{\partial Q_{\mathrm{sp}}}{\partial n_{m}}\right)\right|_{T, V, \mathbf{n}_{m}, \chi}= & \sum_{j=\alpha, \beta} \eta_{m j} \ln \chi_{m j} \\
& -\frac{B_{m}}{8 g Z} \frac{\mathrm{d} g}{\mathrm{~d} \eta} \sum_{i=1}^{c} z_{i} \sum_{j=\alpha, \beta} \eta_{i j} \chi_{i j}\left(\frac{1}{V} \sum_{k=1}^{c} n_{k} \sum_{l=\alpha, \beta} \eta_{k l} \chi_{k l} \delta_{i j, k l}\right) .
\end{aligned}
$$

Substituting 72 gives

$$
\ln \phi_{i}^{\mathrm{assoc}}=\sum_{j=\alpha, \beta} \eta_{i j} \ln \chi_{i j}+\frac{B_{i}}{8 g Z} \frac{\mathrm{d} g}{\mathrm{~d} \eta} \sum_{k=1}^{c} z_{k} \sum_{j=\alpha, \beta} \eta_{k j}\left(\chi_{k j}-1\right) .
$$

Combining (65) and (73), we obtain the full expression for the fugacity coefficient

$$
\begin{aligned}
\ln \phi_{i}=-\ln (Z-B) & +\frac{B_{i}}{B}\left(\frac{B}{Z-B}-\frac{A Z}{Z^{2}+2 B Z-B^{2}}\right) \\
& -\frac{A}{2 \sqrt{2} B}\left(\frac{2 \sum_{j=1}^{c} z_{j} A_{i j}}{A}-\frac{B_{i}}{B}\right) \ln \left[\frac{Z+(1+\sqrt{2}) B}{Z+(1-\sqrt{2}) B}\right] \\
& +\sum_{j=\alpha, \beta} \eta_{i j} \ln \chi_{i j}+\frac{B_{i}}{8 g Z} \frac{\mathrm{d} g}{\mathrm{~d} \eta} \sum_{k=1}^{c} z_{k} \sum_{j=\alpha, \beta} \eta_{k j}\left(\chi_{k j}-1\right) .
\end{aligned}
$$

For completeness, we also present the formula for $\ln \phi_{i}$ in terms of the molar density $\rho$, which can be obtained from (74) using the definitions (36)- 38 and $(66)-(67)$

$$
\begin{aligned}
\ln \phi_{i}=-\ln (1-b \rho)-\ln Z & +\frac{b_{i}}{b}\left(\frac{b \rho}{1-b \rho}-\frac{1}{R T} \frac{a \rho}{1+2 b \rho-(b \rho)^{2}}\right) \\
& -\frac{a}{2 \sqrt{2} b R T}\left(\frac{2 \sum_{j=1}^{c} z_{j} a_{i j}}{a}-\frac{b_{i}}{b}\right) \ln \left[\frac{1+(1+\sqrt{2}) b \rho}{1+(1-\sqrt{2}) b \rho}\right] \\
& +\sum_{j=\alpha, \beta} \eta_{i j} \ln \chi_{i j}+\frac{b_{i} \rho}{8 g} \frac{\mathrm{d} g}{\mathrm{~d} \eta} \sum_{k=1}^{c} z_{k} \sum_{j=\alpha, \beta} \eta_{k j}\left(\chi_{k j}-1\right) .
\end{aligned}
$$

\section{B.4 Pressure and compressibility factor}

The main purpose of this section is to derive the expression for the pressure from the Helmholtz energy departure function. From (61), the pressure is

$$
P=-\left.\left(\frac{\partial F}{\partial V}\right)\right|_{T, \mathbf{n}}
$$

so that if $P^{\mathrm{ig}}=N R T / V$ is the pressure of the ideal gas at $(T, V, \mathbf{n})$,

$$
P-P^{\mathrm{ig}}=-\left.\left(\frac{\partial F^{\text {depart }}}{\partial V}\right)\right|_{T, \mathbf{n}}
$$


As mentioned in Section B.2.3, these derivatives are difficult to evaluate because $F^{\text {depart }}$ in 60 . depends explicitly on pressure, and so the right-hand side will contain explicit volume terms as well as terms which depend on the volume implicitly through the pressure. We can overcome this problem by noting that since all of the mole numbers in these derivatives are held constant, the total number of moles $N$ is also constant. The condition that all mole numbers $\mathbf{n}$ be held constant is equivalent to the condition that all mole fractions $\mathbf{z}$ be held constant. (The converse is not true, however.) We can therefore write the pressure in terms of the molar density $\rho=N / V$ as

$$
P=\left.\frac{\rho^{2}}{N}\left(\frac{\partial F}{\partial \rho}\right)\right|_{T, \mathbf{z}}
$$

The pressure and the compressibility factor $Z=P / \rho R T$ are related to the Helmholtz energy departure function according to

$$
\begin{gathered}
P-P^{\mathrm{ig}}=\left.\frac{\rho^{2}}{N}\left(\frac{\partial F^{\text {depart }}}{\partial \rho}\right)\right|_{T, \mathbf{z}}, \\
\frac{Z-1}{\rho}=\left.\frac{1}{N R T}\left(\frac{\partial F^{\text {depart }}}{\partial \rho}\right)\right|_{T, \mathbf{z}} .
\end{gathered}
$$

These expressions are useful because we can use 51 for $F^{\text {depart }}$, which is the form of $F^{\text {depart }}$ in which $P$ does not appear explicitly.

The pressure is derived from $F^{\text {depart }}$ using 76 . Since $F^{\text {depart }}=F_{\text {phys }}^{\text {depart }}+F_{\text {assoc }}^{\text {depart }}$, we can divide the pressure into a physical contribution (from the PR EOS) and an association contribution, just like we did for the fugacity coefficient in the previous section. The two contributions to $P$ are

$$
\begin{gathered}
P^{\text {phys }}=P^{\text {ig }}+\left.\frac{\rho^{2}}{N}\left(\frac{\partial F_{\text {phys }}^{\text {depart }}}{\partial \rho}\right)\right|_{T, \mathbf{z}}=\rho R T+\left.\frac{\rho^{2}}{N}\left(\frac{\partial F_{\text {phys }}^{\text {depart }}}{\partial \rho}\right)\right|_{T, \mathbf{z}}, \\
P^{\text {assoc }}=\left.\frac{\rho^{2}}{N}\left(\frac{\partial F_{\text {assoc }}^{\text {depart }}}{\partial \rho}\right)\right|_{T, \mathbf{z}} .
\end{gathered}
$$

The derivative of $F_{\text {phys }}^{\text {depart }}$ is

$$
\begin{aligned}
\left.\left(\frac{\partial F_{\text {phys }}^{\text {depart }}}{\partial \rho}\right)\right|_{T, \mathbf{n}} & =\left.N R T \frac{\partial}{\partial \rho}\left(-\ln (1-b \rho)-\frac{a}{2 \sqrt{2} b R T} \ln \left[\frac{1+(1+\sqrt{2}) b \rho}{1+(1-\sqrt{2}) b \rho}\right]\right)\right|_{T, \mathbf{z}} \\
& =N R T\left[\frac{b}{1-b \rho}-\frac{a}{2 \sqrt{2} b R T}\left(\frac{(1+\sqrt{2}) b}{1+(1+\sqrt{2}) b \rho}-\frac{(1-\sqrt{2}) b}{1+(1-\sqrt{2}) b \rho}\right)\right] \\
& =N R T\left[\frac{b}{1-b \rho}-\frac{1}{R T} \frac{a}{1+2 b \rho-(b \rho)^{2}}\right]
\end{aligned}
$$

so that $P^{\text {phys }}$ is 


$$
\begin{aligned}
P^{\text {phys }} & =\rho R T+\rho^{2} R T\left[\frac{b}{1-b \rho}-\frac{1}{R T} \frac{a}{1+2 b \rho-(b \rho)^{2}}\right] \\
& =\frac{\rho R T}{1-b \rho}-\frac{a \rho^{2}}{1+2 b \rho-(b \rho)^{2}} .
\end{aligned}
$$

For the derivative in $(78)$, we encounter the same issue as was encountered with the fugacity coefficient. Namely, we have to evaluate derivatives of the site fractions. We use the same method from Michelsen and Hendriks [53] to overcome this problem. The only difference is that $Q$ depends on density instead of volume and on mole fractions instead of mole numbers. That is, $Q=Q(T, \rho, \mathbf{z}, \chi)$. Let us choose $Q$ to be

$$
\begin{aligned}
Q(T, \rho, \mathbf{z}, \chi)= & \sum_{i=1}^{c} z_{i} \sum_{j=\alpha, \beta} \eta_{i j}\left(\ln \chi_{i j}-\chi_{i j}+1\right) \\
& -\frac{\rho}{2} \sum_{i=1}^{c} z_{i} \sum_{j=\alpha, \beta} \eta_{i j} \chi_{i j} \sum_{k=1}^{c} z_{k} \sum_{l=\alpha, \beta} \eta_{k l} \chi_{k l} \delta_{i j, k l} .
\end{aligned}
$$

It must first be shown that $Q_{\mathrm{sp}}=F_{\text {assoc }}^{\text {depart }} / N R T$. At a stationary point,

$$
\left.\left(\frac{\partial Q_{\mathrm{sp}}}{\partial \chi_{i j}}\right)\right|_{\left(T, \rho, \mathbf{z}, \chi_{i j}\right)}=z_{i} \eta_{i j}\left(\frac{1}{\chi_{i j}}-1\right)-z_{i} \eta_{i j}\left(\rho \sum_{k=1}^{c} z_{k} \sum_{l=\alpha, \beta} \eta_{k l} \chi_{k l} \delta_{i j, k l}\right)=0 .
$$

Rearranging, we have

$$
\rho \sum_{k=1}^{c} z_{k} \sum_{l=\alpha, \beta} \eta_{k l} \chi_{k l} \delta_{i j, k l}=\frac{1}{\chi_{i j}}-1
$$

Substituting this result into 80 and comparing with 40 , we see that

$$
Q_{\mathrm{sp}}=\sum_{i=1}^{c} z_{i} \sum_{j=\alpha, \beta} \eta_{i j}\left(\ln \chi_{i j}-\frac{\chi_{i j}-1}{2}\right)=\frac{F_{\mathrm{assoc}}^{\text {depart }}}{N R T} .
$$

Taking the derivative of 80$)$ and using 81 , we have

$$
\begin{aligned}
& \left.\frac{1}{N R T}\left(\frac{\partial F_{\mathrm{assoc}}^{\text {depart }}}{\partial \rho}\right)\right|_{T, \mathbf{z}}=\left.\left(\frac{\partial Q_{\mathrm{sp}}}{\partial \rho}\right)\right|_{T, \mathbf{z}, \chi} \\
& =\frac{1}{2 \rho} \sum_{i=1}^{c} z_{i} \sum_{j=\alpha, \beta} \eta_{i j}\left(\chi_{i j}-1\right)-\left.\frac{\rho}{2} \sum_{i=1}^{c} z_{i} \sum_{j=\alpha, \beta} \eta_{i j} \chi_{i j} \sum_{k=1}^{c} z_{k} \sum_{l=\alpha, \beta} \eta_{k l} \chi_{k l}\left(\frac{\partial \delta_{i j, k l}}{\partial \rho}\right)\right|_{T, \mathbf{z}, \chi} .
\end{aligned}
$$

Equation 43 shows that the only part of $\delta_{i j, k l}$ that depends on the density is $g$. Therefore, using (48) yields

$$
\left.\left(\frac{\partial \delta_{i j, k l}}{\partial \rho}\right)\right|_{T, \mathbf{z}, \chi}=\left.\frac{\delta_{i j, k l}}{g} \frac{\mathrm{d} g}{\mathrm{~d} \eta}\left(\frac{\partial \eta}{\partial \rho}\right)\right|_{T, \mathbf{z}, \chi}=\frac{\delta_{i j, k l}}{g} \frac{\mathrm{d} g}{\mathrm{~d} \eta} \frac{b}{4}
$$


Substituting into the above, we have

$$
\begin{aligned}
& \left.\frac{1}{N R T}\left(\frac{\partial F_{\mathrm{assoc}}^{\mathrm{depart}}}{\partial \rho}\right)\right|_{T, \mathbf{z}}=\left.\left(\frac{\partial Q_{\mathrm{sp}}}{\partial \rho}\right)\right|_{T, \mathbf{z}, \chi} \\
& =\frac{1}{2 \rho} \sum_{i=1}^{c} z_{i} \sum_{j=\alpha, \beta} \eta_{i j}\left(\chi_{i j}-1\right)-\frac{b}{8 g} \frac{\mathrm{d} g}{\mathrm{~d} \eta} \sum_{i=1}^{c} z_{i} \sum_{j=\alpha, \beta} \eta_{i j} \chi_{i j}\left(\rho \sum_{k=1}^{c} z_{k} \sum_{l=\alpha, \beta} \eta_{k l} \chi_{k l} \delta_{i j, k l}\right) .
\end{aligned}
$$

Substituting (48) and (81) gives

$$
\left.\frac{1}{N R T}\left(\frac{\partial F_{\mathrm{assoc}}^{\mathrm{depart}}}{\partial \rho}\right)\right|_{T, \mathbf{z}}=\left.\left(\frac{\partial Q_{\mathrm{sp}}}{\partial \rho}\right)\right|_{T, \mathbf{z}, \chi}=\frac{1}{2 \rho}\left(1+\frac{\eta}{g} \frac{\mathrm{d} g}{\mathrm{~d} \eta}\right) \sum_{i=1}^{c} z_{i} \sum_{j=\alpha, \beta} \eta_{i j}\left(\chi_{i j}-1\right) .
$$

Multiplying both sides by $\rho^{2} R T$, we get the association contribution to the pressure

$$
P^{\text {assoc }}=\left.\frac{\rho^{2}}{N}\left(\frac{\partial F_{\text {assoc }}^{\text {depart }}}{\partial \rho}\right)\right|_{T, \mathbf{z}}=\frac{\rho R T}{2}\left(1+\frac{\eta}{g} \frac{\mathrm{d} g}{\mathrm{~d} \eta}\right) \sum_{i=1}^{c} z_{i} \sum_{j=\alpha, \beta} \eta_{i j}\left(\chi_{i j}-1\right) .
$$

We combine $(79)$ and $(82)$ to write the complete expression for the pressure

$$
P=\frac{\rho R T}{1-b \rho}-\frac{a \rho^{2}}{1+2 b \rho-(b \rho)^{2}}+\frac{\rho R T}{2}\left(1+\frac{\eta}{g} \frac{\mathrm{d} g}{\mathrm{~d} \eta}\right) \sum_{i=1}^{c} z_{i} \sum_{j=\alpha, \beta} \eta_{i j}\left(\chi_{i j}-1\right) .
$$

Dividing both sides by $\rho R T$, we obtain the compressibility factor

$$
Z=\frac{1}{1-b \rho}-\frac{1}{R T} \frac{a \rho}{1+2 b \rho-(b \rho)^{2}}+\frac{1}{2}\left(1+\frac{\eta}{g} \frac{\mathrm{d} g}{\mathrm{~d} \eta}\right) \sum_{i=1}^{c} z_{i} \sum_{j=\alpha, \beta} \eta_{i j}\left(\chi_{i j}-1\right) .
$$

Using (37)-(38), we can also express the above as

$$
Z=\frac{Z}{Z-B}-\frac{A Z}{Z^{2}+2 B Z-B^{2}}+\frac{1}{2}\left(1+\frac{\eta}{g} \frac{\mathrm{d} g}{\mathrm{~d} \eta}\right) \sum_{i=1}^{c} z_{i} \sum_{j=\alpha, \beta} \eta_{i j}\left(\chi_{i j}-1\right) .
$$

In the absence of associating components, only the first two terms on the right-hand side of (84) are retained. These terms come from the PR EOS. We obtain a cubic polynomial in $Z$ if we rearrange the subsequent equation to have all terms on one side. This result shows why the PR EOS is said to be a cubic equation of state. If the association terms are non-zero, solving for $Z$ becomes more difficult because we do not know a priori the number of roots in (84). As a result, iterative root-finding procedures (usually a combination of the bisection method with Newton's method) must be used over a large search interval such as $Z \in[B+\delta, 1000 B]$, where $\delta$ is a small number. The lower limit in this interval is only slightly larger than $B$ because $B=B(T, P, \mathbf{z})$ represents the closest packing (most compressed state achievable) for a fluid at the conditions $(T, P, \mathbf{z})$. 


\section{B.5 Simplifications employed in the Li and Firoozabadi model}

In addition to the limitation that water is the only self-associating component in the mixture, which we have already discussed in Section B.2.3, Li and Firoozabadi [2] make two more assumptions:

1. All components have a total of four association sites, with two of each type, so that $\eta_{i \alpha}=$ $\eta_{i \beta}=2$ for all components $i$. This four-site model has been shown to work well for water [12].

2. The energetics of association is symmetric between the two types of sites so that $\chi_{i \alpha}=\chi_{i \beta}$ for all $i$. We denote these quantities as $\chi_{i}=\chi_{i \alpha}=\chi_{i \beta}$.

With these simplifications, 40 becomes

$$
\frac{F_{\mathrm{assoc}}^{\text {depart }}}{N R T}=4 \sum_{i=1}^{c} z_{i}\left(\ln \chi_{i}-\frac{\chi_{i}-1}{2}\right) .
$$

As a result, the expressions (51) and 60 for the Helmholtz energy departure function simplify to

$$
\frac{F^{\text {depart }}}{N R T}=-\ln (1-b \rho)-\frac{a}{2 \sqrt{2} b R T} \ln \left[\frac{1+(1+\sqrt{2}) b \rho}{1+(1-\sqrt{2}) b \rho}\right]+4 \sum_{i=1}^{c} z_{i}\left(\ln \chi_{i}-\frac{\chi_{i}-1}{2}\right)
$$

and

$$
\frac{F^{\text {depart }}}{N R T}=\ln \left(\frac{Z}{Z-B}\right)-\frac{A}{2 \sqrt{2} B} \ln \left[\frac{Z+(1+\sqrt{2}) B}{Z+(1-\sqrt{2}) B}\right]+4 \sum_{i=1}^{c} z_{i}\left(\ln \chi_{i}-\frac{\chi_{i}-1}{2}\right),
$$

respectively. The association strengths, cross-association parameters, and site fractions in (53)-(59) are now given by a more tractable set of expressions:

$$
\begin{gathered}
\Delta_{w, w}=g \mathcal{K}\left[\exp \left(\frac{\epsilon}{k_{B} T}\right)-1\right], \\
\Delta_{w, i}=s_{w, i} \Delta_{w, w} \quad i \neq w, \\
\chi_{w}=\frac{Z}{Z+2 \sum_{i=1}^{c} z_{i} \chi_{i} \Delta_{w, i}}, \\
\chi_{i}=\frac{Z}{Z+2 z_{w} \chi_{w} \Delta_{w, i}} .
\end{gathered}
$$

$\mathrm{Li}$ and Firoozabadi have found that fitting to binary water $/ \mathrm{CO}_{2}$ mixture VLE data gives

$$
s_{w, \mathrm{CO}_{2}}=0.0529 T_{r, \mathrm{CO}_{2}}^{2}+0.0404 T_{r, \mathrm{CO}_{2}}-0.0693,
$$

for the cross-association parameter $s_{w, \mathrm{CO}_{2}}$ between water and $\mathrm{CO}_{2}$. Equations (74) and (75) for the fugacity coefficient $\phi_{i}$ become 


$$
\begin{aligned}
& \ln \phi_{i}=-\ln (Z-B)+ \frac{B_{i}}{B}\left(\frac{B}{Z-B}-\frac{A Z}{Z^{2}+2 B Z-B^{2}}\right) \\
&-\frac{A}{2 \sqrt{2} B}\left(\frac{2 \sum_{j=1}^{c} z_{j} A_{i j}}{A}-\frac{B_{i}}{B}\right) \ln \left[\frac{Z+(1+\sqrt{2}) B}{Z+(1-\sqrt{2}) B}\right] \\
&+ 4 \ln \chi_{i}+\frac{B_{i}}{2 g Z} \frac{\mathrm{d} g}{\mathrm{~d} \eta} \sum_{k=1}^{c} z_{k}\left(\chi_{k}-1\right), \\
& \ln \phi_{i}=-\ln (1-b \rho)-\ln Z+\frac{b_{i}}{b}\left(\frac{b \rho}{1-b \rho}-\frac{1}{R T} \frac{a \rho}{1+2 b \rho-(b \rho)^{2}}\right) \\
&-\frac{a}{2 \sqrt{2} b R T}\left(\frac{2 \sum_{j=1}^{c} z_{j} a_{i j}}{a}-\frac{b_{i}}{b}\right) \ln \left[\frac{1+(1+\sqrt{2}) b \rho}{1+(1-\sqrt{2}) b \rho}\right] \\
&+4 \ln \chi_{i}+\frac{b_{i} \rho}{2 g} \frac{\mathrm{d} g}{\mathrm{~d} \eta} \sum_{k=1}^{c} z_{k}\left(\chi_{k}-1\right) .
\end{aligned}
$$

Equations (83)-84) simplify to

$$
\begin{gathered}
P=\frac{\rho R T}{1-b \rho}-\frac{a \rho^{2}}{1+2 b \rho-(b \rho)^{2}}+2 \rho R T\left(1+\frac{\eta}{g} \frac{\mathrm{d} g}{\mathrm{~d} \eta}\right) \sum_{i=1}^{c} z_{i}\left(\chi_{i}-1\right), \\
Z=\frac{1}{1-b \rho}-\frac{1}{R T} \frac{a \rho}{1+2 b \rho-(b \rho)^{2}}+2\left(1+\frac{\eta}{g} \frac{\mathrm{d} g}{\mathrm{~d} \eta}\right) \sum_{i=1}^{c} z_{i}\left(\chi_{i}-1\right), \\
Z=\frac{Z}{Z-B}-\frac{A Z}{Z^{2}+2 B Z-B^{2}}+2\left(1+\frac{\eta}{g} \frac{\mathrm{d} g}{\mathrm{~d} \eta}\right) \sum_{i=1}^{c} z_{i}\left(\chi_{i}-1\right) .
\end{gathered}
$$

The enthalpy results presented in Sections 2 and 3 are calculated using the equations presented in this section. As we demonstrate in those sections, this simplified version of the CPA EOS is sufficient for molar enthalpy calculations of mixtures containing $\mathrm{CO}_{2}$ and $\mathrm{H}_{2} \mathrm{O}$, as well as enthalpies of pure components. It has previously been shown to provide excellent agreement with VLE (solubility) and density data [2]. It cannot, however, accurately calculate excess enthalpies of $\mathrm{CO}_{2}-\mathrm{H}_{2} \mathrm{O}$ mixtures. In Section 4, we reason why it fails for excess enthalpy calculations and explain how relaxing the two assumptions stated in this section, so that the equations in Sections B.3 and B.4 are followed instead, may allow for better agreement with experimental excess enthalpy data.

\section{Duan and Sun $\mathrm{CO}_{2}$ activity coefficient model}

In electrolytic solutions, concentrations are usually expressed in terms of molality $(m)$ rather than mole fractions, so that 21 - 23 must be recast in terms of molality. In the molality convention, a natural choice for the activity coefficient $\gamma_{i}$ of a solute species $i$ is

$$
\lim _{m_{i} \rightarrow 1} \gamma_{i}=1
$$

With $\gamma_{i}$ defined in this way, the activity $\mathrm{a}_{i}$ and chemical potential $\mu_{i}$ of $i$ are given by 


$$
\begin{gathered}
\mathrm{a}_{i}=\gamma_{i} m_{i}=\frac{f_{i}\left(T, P, m_{i}\right)}{f_{i}^{\mathrm{im}}\left(T, P, m_{i}=1\right)} . \\
\mu_{i}\left(T, P, m_{i}\right)-\mu_{i}^{\mathrm{im}}\left(T, P, m_{i}=1\right)=R T \ln \mathrm{a}_{i} .
\end{gathered}
$$

Here, $f_{i}^{\mathrm{im}}\left(T, P, m_{i}=1\right)$ and $\mu_{i}^{\mathrm{im}}\left(T, P, m_{i}=1\right)$ are the fugacity and chemical potential of $i$, respectively, in an ideal mixture where $m_{i}=1$. These equations apply to the aqueous phase, which is the electrolytic phase. For the $\mathrm{CO}_{2}$-rich phase, which is assumed to contain only $\mathrm{CO}_{2}$ and water, Duan and Sun [3] choose the reference chemical potential to be that of the pure component in the ideal gas state at a pressure $P^{\prime}=1$ bar. Using (12)-(15), the chemical potential $\mu_{i}(T, P, \mathbf{z})$ of component $i$ in the $\mathrm{CO}_{2}$-rich phase, with pressure expressed in units of bars, is

$$
\mu_{i}(T, P, \mathbf{z})=\mu_{i}^{\mathrm{ig}}\left(T, P^{\prime}=1 \text { bar }\right)+R T \ln z_{i} P+R T \ln \phi_{i}(T, P, \mathbf{z}) .
$$

For pure $\mathrm{CO}_{2}$, this equation simplifies to

$$
\mu_{\mathrm{CO}_{2}}(T, P)=\mu_{\mathrm{CO}_{2}}^{\mathrm{ig}}\left(T, P^{\prime}=1 \text { bar }\right)+R T \ln P+R T \ln \phi_{\mathrm{CO}_{2}}(T, P) .
$$

Substituting (28) and (29) into the definition (5) of the $\mathrm{CO}_{2}$ molar enthalpy of solution $\Delta h_{\text {sol }}$,

$$
\begin{aligned}
\Delta h_{\mathrm{sol}}= & \bar{H}_{\mathrm{CO}_{2}}\left(T, P, m_{\mathrm{CO}_{2}} \rightarrow 0\right)-h_{\mathrm{CO}_{2}}(T, P) \\
& =-T^{2} \frac{\partial}{\partial T}\left[\left.\left(\frac{\mu_{\mathrm{CO}_{2}}\left(T, P, m_{\mathrm{CO}_{2}} \rightarrow 0\right)}{T}\right)\right|_{P, \mathbf{m}}-\left.\left(\frac{\mu_{\mathrm{CO}_{2}}(T, P)}{T}\right)\right|_{P}\right] .
\end{aligned}
$$

The subscript $\mathbf{m}$ indicates that all species concentrations are held fixed. From (86) and (87),

$$
\begin{gathered}
\left.\frac{\partial}{\partial T}\left(\frac{\mu_{\mathrm{CO}_{2}}\left(T, P, m_{\mathrm{CO}_{2}} \rightarrow 0\right)}{T}\right)\right|_{P, \mathbf{m}}= \\
\left.\frac{\partial}{\partial T}\left(\frac{\mu_{\mathrm{CO}_{2}}^{\mathrm{im}}\left(T, P, m_{\mathrm{CO}_{2}}=1\right)}{T}\right)\right|_{P} \\
+\left.R\left(\frac{\partial \ln \gamma_{\mathrm{CO}_{2}}\left(T, P, m_{\mathrm{CO}_{2}} \rightarrow 0\right)}{\partial T}\right)\right|_{P, \mathbf{m}}, \\
\left.\frac{\partial}{\partial T}\left(\frac{\mu_{\mathrm{CO}_{2}}(T, P)}{T}\right)\right|_{P}=\left.\frac{\partial}{\partial T}\left(\frac{\mu_{\mathrm{CO}_{2}}^{\mathrm{ig}}\left(T, P^{\prime}=1 \mathrm{bar}\right)}{T}\right)\right|_{P}+\left.R\left(\frac{\partial \ln \phi_{\mathrm{CO}_{2}}(T, P)}{\partial T}\right)\right|_{P} .
\end{gathered}
$$

For a brine solution where the only electrolyte is $\mathrm{NaCl}$, Duan and Sun's $\mathrm{CO}_{2}$ activity coefficient model is

$$
\ln \gamma_{\mathrm{CO}_{2}}=2 \lambda_{\mathrm{CO}_{2}-\mathrm{Na}} m_{\mathrm{Na}}+2 \lambda_{\mathrm{CO}_{2}-\mathrm{Cl}} m_{\mathrm{Cl}}+\zeta_{\mathrm{CO}_{2}-\mathrm{Na}-\mathrm{Cl}} m_{\mathrm{Na}} m_{\mathrm{Cl}} .
$$

The coefficients $\lambda_{\mathrm{CO}_{2}-\mathrm{Na}}, \lambda_{\mathrm{CO}_{2}-\mathrm{Cl}}, \zeta_{\mathrm{CO}_{2}-\mathrm{Na}-\mathrm{Cl}}$ are temperature- and pressure-dependent functions that represent intermolecular interactions between $\mathrm{CO}_{2}$ and the dissolved ions. Equation (88) is obtained by taking the derivative, with respect to the molality of $\mathrm{CO}_{2}$, of a Pitzer-type excess Gibbs energy function [38. Duan and Sun treat $\mu_{\mathrm{CO}_{2}}^{\mathrm{ig}}\left(T, P^{\prime}=1\right.$ bar $)$ and $\lambda_{\mathrm{CO}_{2}-\mathrm{Cl}}$ as being identically equal to zero. Using many sources of $\mathrm{CO}_{2}$ solubility data in pure water and $\mathrm{NaCl}$ solutions, they fit the functions $\mu_{\mathrm{CO}_{2}}^{\mathrm{im}}\left(T, P, m_{\mathrm{CO}_{2}}=1\right) / R T, \lambda_{\mathrm{CO}_{2}-\mathrm{Na}}$, and $\zeta_{\mathrm{CO}_{2}-\mathrm{Na}-\mathrm{Cl}}$ to expressions of the form 


$$
c_{1}+c_{2} T+\frac{c_{3}}{T}+c_{4} T^{2}+\frac{c_{5}}{630-T}+c_{6} P+c_{7} P \ln T+\frac{c_{8} P}{T}+\frac{c_{9} P}{630-T}+\frac{c_{10} P^{2}}{(630-T)^{2}}+c_{11} T \ln P,
$$

where the $c$ 's are constant coefficients. The fugacity coefficient $\phi_{\mathrm{CO}_{2}}(T, P)$ is obtained by iteratively solving a nonlinear equation, as described in their paper. In a later study, Duan et al. present a series of piecewise expressions (curve fits) for $\phi_{\mathrm{CO}_{2}}(T, P)$ so that it can be calculated directly, without the need for iterative methods [54]. Although these expressions may increase the efficiency of $\mathrm{CO}_{2}$ solubility computations, they cannot be used for enthalpy computations. We have found that for certain conditions, they lead to sharp jumps in the enthalpy. This unphysical behavior occurs because the enthalpy is obtained from the fugacity coefficient by taking a temperature derivative, so that it should be represented by a smooth function of temperature, rather than a series of piecewise expressions. We therefore compute $\phi_{\mathrm{CO}_{2}}(T, P)$ as described in the earlier study.

\section{References}

[1] N. Spycher, K. Pruess, A model for thermophysical properties of $\mathrm{CO}_{2}$-brine mixtures at elevated temperatures and pressures, in: Proceedings of the Thirty-Sixth Workshop on Geothermal Reservoir Engineering, Stanford University, 2011. URL https://pangea.stanford.edu/ERE/db/IGAstandard/record_detail.php?id=7167

[2] Z. Li, A. Firoozabadi, Cubic-plus-association equation of state for water-containing mixtures: Is "cross association" necessary?, AIChE J. 55 (7) (2009) 1803-1813. doi:10.1002/aic.11784

[3] Z. Duan, R. Sun, An improved model calculating $\mathrm{CO}_{2}$ solubility in pure water and aqueous $\mathrm{NaCl}$ solutions from 273 to $533 \mathrm{~K}$ and from 0 to 2000 bar, Chem. Geol. 193 (2003) 257-271. doi:10.1016/S0009-2541(02)00263-2.

[4] N. Spycher, K. Pruess, J. Ennis-King, $\mathrm{CO}_{2}-\mathrm{H}_{2} \mathrm{O}$ mixtures in the geological sequestration of $\mathrm{CO}_{2}$. I. Assessment and calculation of mutual solubilities from 12 to $100^{\circ} \mathrm{C}$ and up to $600 \mathrm{bar}$, Geochim. Cosmochim. Acta 67 (2003) 3015-3031. doi:10.1016/S0016-7037(03)00273-4.

[5] P. C. Myint, A. Firoozabadi, Onset of convection with fluid compressibility and interface movement, Phys. Fluids 25 (2013) 094105. doi:10.1063/1.4821743.

[6] M. M. Smith, Y. Sholokhova, Y. Hao, S. A. Carroll, $\mathrm{CO}_{2}$-induced dissolution of low permeability carbonates. Part I: Characterization and experiments, Adv. Water Resour. 62 (2013) 370-387. doi:10.1016/j .advwatres.2013.09.008.

[7] Y. Hao, M. M. Smith, Y. Sholokhova, S. A. Carroll, $\mathrm{CO}_{2}$-induced dissolution of low permeability carbonates. Part II: Numerical modeling of experiments, Adv. Water Resour. 62 (2013) 388-408. doi:10.1016/j.advwatres.2013.09.009.

[8] M. T. Elenius, J. M. Nordbotten, H. Kalisch, Convective mixing influenced by the capillary transition zone, Comput. Geosci. 18 (2014) 417-431. doi:10.1007/s10596-014-9415-1.

[9] A. C. Slim, Solutal-convection regimes in a two-dimensional porous medium, J. Fluid Mech. 741 (2014) 461-491. doi:10.1017/jfm.2013.673. 
[10] V. Loodts, L. Rongy, A. De Wit, Impact of pressure, salt concentration, and temperature on the convective dissolution of carbon dioxide in aqueous solutions, Chaos 24 (2014) 043120. doi:10.1063/1.4896974.

[11] National Risk Assessment Partnership (NRAP). URL http://esd.lbl.gov/research/programs/gcs/projects/nrap/

[12] G. M. Kontogeorgis, M. L. Michelsen, G. K. Folas, S. Derawi, N. von Solms, E. H. Stenby, Ten years with the CPA (Cubic-Plus-Association) equation of state. Part 2. Cross-associating and multicomponent systems, Ind. Eng. Chem. Res. 45 (2006) 4869-4878. doi:10.1021/ ie051306n.

[13] J. Moortgat, Z. Li, A. Firoozabadi, Three-phase compositional modeling of $\mathrm{CO}_{2}$ injection by higher-order finite element methods with CPA equation of state for aqueous phase, Water Resour. Res. 48 (2012) W12511. doi:10.1029/2011WR011736.

[14] J. M. Smith, H. C. Van Ness, M. M. Abbott, Introduction to Chemical Engineering Thermodynamics, McGraw-Hill, New York, 2004.

[15] National Institute of Standards and Technology (NIST), Chemistry WebBook: Thermophysical properties of fluid systems

URL http://webbook.nist.gov/chemistry/fluid/

[16] J. R. Cooper, Representation of the ideal-gas thermodynamic properties of water, Int. J. Thermophys. 3 (1982) 35-43. doi:10.1007/BF00503957.

[17] A. Firoozabadi, P. Cheng, Prospects for subsurface $\mathrm{CO}_{2}$ sequestration, AIChE J. 56 (6) (2010) 1398-1405. doi:10.1002/aic.12287.

[18] M. R. Patel, P. T. Eubank, Experimental densities and derived thermodynamic properties for carbon dioxide-water mixtures, J. Chem. Eng. Data 33 (1988) 185-193. doi:10.1021/ je00052a037.

[19] D. Koschel, J.-Y. Coxam, L. Rodier, V. Majer, Enthalpy and solubility data of $\mathrm{CO}_{2}$ in water and $\mathrm{NaCl}(\mathrm{aq})$ at conditions of interest for geological sequestration, Fluid Phase Equilibr. 247 (2006) 107-120. doi:10.1016/j.fluid.2006.06.006.

[20] E. E. Michaelides, Thermodynamic properties of geothermal fluids, in: Geothermal Resources Council Annual Meeting Transactions, Vol. 5, Houston, TX, USA, 1981, pp. 361-364.

[21] J.-S. Gudmundsson, H. Thráinsson, Power potential of two-phase geothermal wells, Geothermics 18 (1989) 357-366. doi:10.1016/0375-6505(89)90062-X.

[22] S. B. Bottini, G. Saville, Excess enthalpies for (water + nitrogen)(g) and (water + carbon dioxide)(g) at 520 to $620 \mathrm{~K}$ and up to $4.5 \mathrm{MPa}$, J. Chem. Thermodyn. 17 (1985) 83-97. doi:10.1016/0021-9614(85)90034-5.

[23] J. S. Gallagher, R. Crovetto, J. M. H. Levelt Sengers, The thermodynamic behavior of the $\mathrm{CO}_{2}-\mathrm{H}_{2} \mathrm{O}$ system from 400 to $1000 \mathrm{~K}$, up to $100 \mathrm{MPa}$ and $30 \%$ mole fraction of $\mathrm{CO}_{2}$, J. Phys. Chem. Ref. Data 22 (1993) 431-513. doi:10.1063/1.555938. 
[24] C. J. Wormald, N. M. Lancaster, A. J. Sellars, The excess molar enthalpies of $x \mathrm{H}_{2} \mathrm{O}+(1-$ $x) \mathrm{CO}(\mathrm{g})$ and $x \mathrm{H}_{2} \mathrm{O}+(1-x) \mathrm{CO}_{2}(\mathrm{~g})$ at high temperatures and pressures, J. Chem. Thermodyn. 18 (1986) 135-147. doi:10.1016/0021-9614(86)90128-X.

[25] G. M. Wilson, C. J. Brady, Heats of mixing of steam with $\mathrm{N}_{2}, \mathrm{CO}_{2}, \mathrm{H}_{2}, \mathrm{CH}_{4}$, and $\mathrm{CO}$ at high temperatures and pressures using a new high temperature calorimeter (RR-73), Tech. rep., Gas Processors Association (1983).

[26] A. Burrows, J. Holman, A. Parsons, G. Pilling, G. Price, Chemistry ${ }^{3}$ : Introducing inorganic, organic, and physical chemistry, 2nd Edition, Oxford University Press, Oxford, 2013.

[27] W. Yan, S. Huang, E. H. Stenby, Measurement and modeling of $\mathrm{CO}_{2}$ solubility in $\mathrm{NaCl}$ brine and $\mathrm{CO}_{2}$-saturated $\mathrm{NaCl}$ brine density, Int. J. Greenh. Gas Con. 5 (2011) 1460-1477. doi: $10.1016 /$ j.ijggc.2011.08.004

[28] Z. Duan, J. Hu, D. Li, S. Mao, Densities of the $\mathrm{CO}_{2}-\mathrm{H}_{2} \mathrm{O}$ and $\mathrm{CO}_{2}-\mathrm{H}_{2} \mathrm{O}-\mathrm{NaCl}$ systems up to $647 \mathrm{~K}$ and $100 \mathrm{MPa}$, Energy \& Fuels 22 (2008) 1666-1674. doi:10.1021/ef700666b.

[29] M. A. Nomeli, N. Tilton, A. Riaz, A new model for the density of saturated solutions of $\mathrm{CO}_{2}{ }^{-}$ $\mathrm{H}_{2} \mathrm{O}-\mathrm{NaCl}$ in saline aquifers, Int. J. Greenh. Gas Con. 31 (2014) 192-204. doi:10.1016/j. ijggc.2014.10.006.

[30] A. H. Harvey, J. M. Prausnitz, Thermodynamics of high-pressure aqueous systems containing gases and salts, AIChE J. 35 (1989) 635-644. doi:10.1002/aic.690350413.

[31] J. A. Myers, S. I. Sandler, R. H. Wood, An equation of state for electrolyte solutions covering wide ranges of temperature, pressure, and composition, Ind. Eng. Chem. Res. 41 (2002) 32823297. doi:10.1021/ie011016g.

[32] Y. Lin, K. Thomsen, J.-C. de Hemptinne, Multicomponent equations of state for electrolytes, AIChE J. 53 (2007) 989-1005. doi:10.1002/aic.11128.

[33] S. I. Sandler, Chemical, Biochemical, and Engineering Thermodynamics, 4th Edition, John Wiley \& Sons, Hoboken, 2006.

[34] J. M. Prausnitz, R. N. Lichtenthaler, E. Gomez de Azevedo, Molecular Thermodynamics of Fluid-Phase Equilibria, 3rd Edition, Prentice Hall, Upper Saddle River, 1999.

[35] M. L. Michelsen, J. M. Mollerup, Thermodynamic Models: Fundamentals and Computational Aspects, 2nd Edition, Tie-Line Publications, Rønnebaervej, 2007.

[36] A. Firoozabadi, Thermodynamics and Application to Hydrocarbon Energy Production, McGraw-Hill, New York, 2015.

[37] J. R. Elliott, C. T. Lira, Introductory Chemical Engineering Thermodynamics, 1st Edition, Prentice Hall, Upper Saddle River, 1999.

[38] K. S. Pitzer, Thermodynamics of electrolytes. I. Theoretical basis and general equations, J. Phys. Chem. 77 (1973) 268-277. doi:10.1021/j100621a026. 
[39] B. Widom, The non-ideal gas, Cambridge University Press, Cambridge, 2002, Ch. 6 of Statistical Mechanics: A Concise Introduction for Chemists, pp. 81-100.

[40] G. M. Kontogeorgis, E. C. Voutsas, I. V. Yakoumis, D. P. Tassios, An equation of state for associating fluids, Ind. Eng. Chem. Res. 35 (1996) 4310-4318. doi:10.1021/ie9600203.

[41] J. R. Elliott, S. J. Suresh, M. D. Donohue, A simple equation of state for non-spherical and associating molecules, Ind. Eng. Chem. Res. 29 (1990) 1476-1485. doi:10.1021/ie00103a057

[42] I. G. Economou, C. Tsonopoulos, Associating models and mixing rules in equations of state for water/hydrocarbon mixtures, Chem. Eng. Sci. 52 (1997) 511-525. doi:10.1016/ S0009-2509(96)00441-1.

[43] G. M. Kontogeorgis, G. K. Folas, Thermodynamic Models for Industrial Applications: From Classical and Advanced Mixing Rules to Association Theories, 1st Edition, John Wiley \& Sons, Chichester, 2010.

[44] M. S. Wertheim, Fluids with highly directional attractive forces. I. Statistical thermodynamics, J. Stat. Phys. 35 (1984) 19-34. doi:10.1007/BF01017362.

[45] M. S. Wertheim, Fluids with highly directional attractive forces. II. Thermodynamic perturbation theory and integral equations, J. Stat. Phys. 35 (1984) 35-47. doi : 10.1007/BF01017363

[46] M. S. Wertheim, Fluids with highly directional attractive forces. III. Multiple attraction sites, J. Stat. Phys. 42 (1986) 459-476. doi:10.1007/BF01127721.

[47] M. S. Wertheim, Thermodynamic perturbation theory of polymerization, J. Chem. Phys. 87 (1987) 7323. doi:10.1063/1.453326.

[48] G. M. Kontogeorgis, M. L. Michelsen, G. K. Folas, S. Derawi, N. von Solms, E. H. Stenby, Ten years with the CPA (Cubic-Plus-Association) equation of state. Part 1. Pure compounds and self-associating systems, Ind. Eng. Chem. Res. 45 (2006) 4855-4868. doi :10.1021/ie051305v

[49] Z. Li, A. Firoozabadi, Cubic-plus-association equation of state for asphaltene precipitation in live oils, Energy \& Fuels 24 (2010) 2956-2963. doi:10.1021/ef9014263.

[50] Z. Li, A. Firoozabadi, Modeling asphaltene precipitation by n-alkanes from heavy oils and bitumens using cubic-plus-association equation of state, Energy \& Fuels 24 (2010) 1106-1113. doi:10.1021/ef9009857.

[51] A. Firoozabadi, Thermodynamics of Hydrocarbon Reservoirs, McGraw-Hill, New York, 1999.

[52] P. M. Mathias, T. Naheiri, E. M. Oh, A density correction for the Peng-Robinson equation of states, Fluid Phase Equilibr. 47 (1989) 77-87. doi:10.1016/0378-3812(89)80051-2.

[53] M. L. Michelsen, E. M. Hendriks, Physical properties from association models, Fluid Phase Equilibr. 180 (2001) 165-174. doi:10.1016/S0378-3812(01)00344-2.

[54] Z. Duan, R. Sun, C. Zhu, I.-M. Chou, An improved model for the calculation of $\mathrm{CO}_{2}$ solubility in aqueous solutions containing $\mathrm{Na}^{+}, \mathrm{K}^{+}, \mathrm{Ca}^{2+}, \mathrm{Mg}^{2+}, \mathrm{Cl}^{-}$, and $\mathrm{SO}_{4}^{2-}$, Mar. Chem. 98 (2006) 131-139. doi:10.1016/j.marchem.2005.09.001. 